\title{
Optically Active Nanomaterials for Bioimaging and Targeted Therapy
}

\author{
Yu Yang ${ }^{1 *}$, Li Wang ${ }^{1,2}$, Bin Wan ${ }^{1,2}$, Yuxin $\mathrm{Gu}^{1,2}$ and Xinxin $\mathrm{Li}^{3}$ \\ ${ }^{1}$ State Key Laboratory of Environmental Chemistry and Ecotoxicology, Research Center for Eco-Environmental Sciences, \\ Chinese Academy of Sciences, Beijing, China, ${ }^{2}$ College of Resources and Environment, University of Chinese Academy of \\ Sciences, Beijing, China, ${ }^{3}$ Rural Energy and Environment Agency, Ministry of Agriculture, Beijing, China
}

\section{OPEN ACCESS}

Edited by:

Yu Gao,

Nanjing University of Posts and

Telecommunications, China

Reviewed by:

Shuai Yu,

University of Texas at Arlington,

United States

Xinyi Wang,

Shenyang Agricultural

University, China

${ }^{*}$ Correspondence:

Yu Yang

yuyang@rcees.ac.cn

Specialty section:

This article was submitted to

Nanobiotechnology,

a section of the journal

Frontiers in Bioengineering and

Biotechnology

Received: 05 August 2019

Accepted: 25 October 2019

Published: 15 November 2019

Citation:

Yang Y, Wang L, Wan B, Gu Y and Li X (2019) Optically Active Nanomaterials for Bioimaging and Targeted Therapy.

Front. Bioeng. Biotechnol. 7:320.

doi: 10.3389/fbioe.2019.00320
Non-invasive tracking for monitoring the selective delivery and transplantation of biotargeted agents in vivo has been employed as one of the most effective tools in the field of nanomedicine. Different nanoprobes have been developed and applied to bioimaging tissues and the treatment of diseases ranging from inflammatory and cardiovascular diseases to cancer. Herein, we will review the recent advances in the development of optics-responsive nanomaterials, including organic and inorganic nanoparticles, for multimodal bioimaging and targeted therapy. The main focus is placed on nanoprobe fabrication, mechanistic illustrations, and diagnostic, or therapeutical applications. These nanomedicine strategies have promoted a better understanding of the biological events underlying diverse disease etiologies, thereby facilitating diagnosis, illness evaluation, therapeutic effect, and drug discovery.

Keywords: optical, nanomaterials, bioimaging, tissues, therapy

\section{INTRODUCTION}

Molecular imaging reflects biological information on temporal and spatial scales, unveiling the dynamics of disease (Smith and Gambhir, 2017). It is a crucial diagnostic tool for monitoring in vivo response and assessing outcomes in targeted therapies (Fan et al., 2017). Also, integrating imaging, and therapy into theranostic systems is an efficient strategy for real-time tracking of the pharmacokinetics and biodistribution of a drug. Current imaging modalities include X-ray, magnetic resonance, optics (e.g., fluorescence, luminescence, Raman, photoacoustics), radionuclides, and mass spectrometry (Kunjachan et al., 2015). Among them, optical imaging is a common modality in preclinical research on theranostic agents.

Nanomaterials have been widely developed as therapeutic and diagnostic agents (Lim et al., 2015; Chen et al., 2016a). Research efforts have changed from developing new materials in vitro to exploring functional materials in vivo, thereby increasing the potential for clinical translation (Lamch et al., 2018). Perhaps the unique advantage of nanoparticles (NPs) for optical imaging is that their physical and optical properties are easily tunable through structural modulation. Also, some NP compositions possess inherent imaging and therapeutic properties, while others are rendered multifunctional through the manipulation of multiple structural elements. Engineering multifunctional theranostic NPs presents several challenges, including imaging quality, loading capacity, the toxicity of intrinsic ingredients, storage, and in vivo stability, the complexity of synthesis, batch repeatability, production costs, and regulatory hurdles (Farokhzad and Langer, 2006; Lee et al., 2012). Common nanomaterials, including inorganic and organic NPs, have demonstrated a potential for diagnosis and therapy (Brigger et al., 2002). Variations in size, shape, and surface modifications can adjust their biocompatibility and specificity with target tissues 
(Wang and Thanou, 2010). Depending on their structural composition, NPs can provide an optical signal or function as nanocarriers for optically active agents. Current interests mainly involve non-invasive imaging of deep tissues and targeting drug therapy.

In this paper, we discuss recent progress in optical-sensitive NPs, their bioimaging involving fluorescence, luminescence, surface-enhanced Raman scattering (SERS), and photoacoustic (PA) signals, and their therapeutic applications in photodynamic therapy (PDT), photothermal therapy (PTT), and drug delivery. Moreover, common design considerations for advanced nanomedicines and the challenges of their application are discussed from diagnostic and therapeutic perspectives.

\section{OPTICALLY ACTIVE NANOMATERIALS}

\section{Inorganic Nanomaterials}

Due to their unique characteristics, i.e., surface plasmon resonance (SPR), gold NPs (GNPs) are usually chosen to enhance optical imaging based on their absorption, fluorescence, Raman scattering, etc. (Wu et al., 2019). Generally, GNPs are synthesized by $\mathrm{HAuCl}_{4}$ reduction, known as the Brust et al. (1994) or Turkevich method Turkevich et al. (1951). GNPs are stabilized by a wide variety of ligands that affect their sizes and properties (Treguer-Delapierre et al., 2008; Boisselier et al., 2010). Their diameters range from $1 \mathrm{~nm}$ to more than $120 \mathrm{~nm}$. Also, diverse shapes can be prepared, such as core-shell nanostructures (Kharlamov et al., 2015), nanorods (de la Zerda et al., 2015), or nanocages (Chen et al., 2005a) whose aspect ratios modulate their optical properties. The excellent stability of GNPs covalently bonded with thiolated ligands permits chemical modifications directly on their surfaces (Boisselier et al., 2008). The ligands for stabilizing GNPs can be specifically selected for drug encapsulation and release or targeted to tissues such as tumors (Guo et al., 2017; Her et al., 2017; Spyratou et al., 2017). However, the safety of GNPs in clinical application remains controversial, with more information required on their longterm toxicity in vivo.

Carbon-based nanomaterials $(\mathrm{CBN})$ as members of the carbon family including fullerenes, carbon nanotubes (CNTs), graphene $(\mathrm{G})$, graphene oxide $(\mathrm{GO})$, nanodiamonds (NDDs), and carbon dots (CDs) (Bartelmess et al., 2015; Patel et al., 2019). Modifying their surfaces with functional groups (e.g., carboxylic acid, hydroxyl, or epoxy) provides the opportunity to optimize their properties. The extraordinary optical features of CBN (e.g., inherent fluorescence, high photostability, and tunable narrow emission spectra) increase their potential for imaging and diagnosis of cells or tissues (Sadegh and Shahryari-ghoshekandi, 2015; Goodarzi et al., 2017; Namdari et al., 2017; Bullock and Bussy, 2019; Tinwala and Wairkar, 2019). Fullerenes usually act as photosensitizers (PSs) to generate singlet oxygen $\left({ }^{1} \mathrm{O}_{2}\right)$ and hence are applied for blood sterilization and cancer PDT (Lu et al., 2019). The inherent spectroscopic features of CNTs (e.g., Raman scattering and photoluminescence) provide a valuable means of tracking in vivo therapeutic status, pharmacodynamic behavior, and drug delivery efficiency and imaging and detecting diseases (Tasis et al., 2006; Liu et al., 2011). Graphene and
GO-based nanocarriers have attracted significant attention for imaging and anticancer therapy because of their large drug loading and effective delivery capacity. Also, $\sim 2,600 \mathrm{~m}^{2} / \mathrm{g}$ is more than double the surface area of most nanomaterials (Mao et al., 2013; Reina et al., 2017). Recently, carbon dots (CDs, size < $10 \mathrm{~nm}$ ) have been extensively studied to gain a high fluorescence quantum yield through facile synthesis methods (Liu et al., 2015a). NDDs are nanocrystals that consist of tetrahedrally bonded carbon atoms in the form of a three-dimensional (3D) cubic lattice. The optical properties of NDDs allow their use as photoluminescent probes $\left(\lambda_{\mathrm{em}}=550-800 \mathrm{~nm}\right)$ due to nitrogenvacancy defect centers (Chang et al., 2008). When functionalized, their biocompatibility is known to be superior to CNTs and carbon black (Mochalin et al., 2013). However, the toxicity of $\mathrm{CBN}$ is presently the key problem for their clinical use. Also, the toxicology and pharmacokinetics of $\mathrm{CBN}$ mainly rely on several factors, e.g., physicochemical and structural properties, exposure dose and time, cell type, mechanism, residual catalyst, and synthesis method. It is necessary to systematically evaluate CBN in vivo safety using more relevant animal models.

Porous silicon nanoparticles ( $\mathrm{pSiNPs}$ ) have gained intense attention in the biomedical field due to their low toxicity and potential for use in minimally invasive and focal therapies that avoid conventional side effects (Vivero-Escoto et al., 2012). pSiNPs can be degraded completely to produce non-toxic orthosilicic acid (Tzur-Balter et al., 2013), a bioavailable form of $\mathrm{Si}$, and then excreted efficiently through renal clearance (Park et al., 2009). Also, their high active surface areas (up to $1,000 \mathrm{~m}^{2} / \mathrm{g}$ ) (Loni et al., 2015) allow Si modifications by other molecules through various surface chemical reactions (e.g., hydrosilylation, silanisation, and hydrocarbonization), which facilitate their targeted and controlled drug release into cancer cells (Wu et al., 2011a; Makila et al., 2012; Wang et al., 2014). Actually, the porous nanostructures of pSiNPs allow them to load drugs, probes, enzymes, proteins, antibodies, siRNA, or other species (Castillo and Vallet-Regi, 2019). Numerous cell-specific epitopes and biomarkers afford selective binding to certain antibodies, peptides, or other molecules and thus provide the opportunity for targeted drug delivery via vectorized pSiNPs (Li et al., 2018a). Moreover, the optical features of silicon nanostructures, i.e., intrinsic photoluminescence, afford an alternative for bioimaging, accompanied with better biocompatibility and biodegradability and lower toxicity compared with semiconductor quantum dots (Warner et al., 2005; Erogbogbo et al., 2008; Bimbo et al., 2010; Gu et al., 2010). Finally, easy handling and cost-efficient preparation through facile electrochemical anodization of crystalline silicon increase the interest in utilizing pSiNPs (Sailor, 2012). Nevertheless, the design and synthesis of smart multifunctional pSiNP nanocarriers, in vivo performance evaluation using animal models, and subsequent translational studies still need to be further explored.

Lanthanide (Ln)-doped upconversion nanoparticles (UCNPs) can convert two or more low-energy near-infrared (NIR) photons into high-energy emissions through a non-linear antiStokes process (Auzel, 2004). Due to their special upconversion luminescence feature and stable Ln-based inorganic framework, 
Ln-UCNPs have been developed as promising alternatives to conventional labels (e.g., organic dyes and quantum dots) with several attractive advantages, such as being highly resistant to photobleaching and photoblinking, having negligible background autofluorescence, having deeper tissue-penetration ability, and causing minimal photodamage (Zhou et al., 2012). Also, considering the high feasibility and maneuverability of their structural design, many functional components with other imaging capabilities are introduced into these nanoparticles for diagnostic and therapeutic applications (Liu et al., 2014a, 2015b). Likewise, UCNPs with a high surface area, characteristic structures, and versatile surface functionalization can act as nanocarriers for drug delivery (Wu et al., 2015; Yang et al., 2015a). Significantly, UCNPs with a distinctive upconverted emission can double as light-transducers to activate PSs for deep-tissue PDT or as phototriggered devices for precise control of drug-payload release induced by NIR light (Wang et al., 2013a; Min et al., 2014; Idris et al., 2015). However, high upconversion efficiency is realized only upon 980- or 808-nm excitation, which has seriously hindered their practical usage in the biomedical field. Also, surface modification and bioconjugation of long-term stable and biocompatible Ln-UCNPs remain a huge challenge. Therefore, more strategies for surface modification and corresponding toxicology studies of these NPs are still needed for future preclinical and clinical applications.

Quantum dots (QDs) are semiconductor nanocrystals (typically with diameters $<10 \mathrm{~nm}$ ), which display dimensiondominant optical features (e.g., absorption and luminescence) (Xu et al., 2018a). Particularly, QDs of various dimensions or ingredients are excited by a single light source to separately emit diverse colors over a wide range with negligible spectral overlap, rendering them desirable for multiple imaging (Yong, 2012). Avoiding the photobleaching of organic dyes, QDs are more beneficial to imaging in vivo for a long time (Geszke-Moritz and Moritz, 2013). Significantly, QDs can be tuned to emit over a broad wavelength range (e.g., $450-1,500 \mathrm{~nm}$ ) by modulating their dimensions, configurations, and components (Pichaandi and van Veggel, 2014; Zhao et al., 2018). QDs can also be conjugated with a recognition molecule, such as antibodies, DNA, proteins, etc. (Lu and Li, 2011). Flexibility in the surface chemistry and emission peak of QDs makes them useable as optical probes or nanocarriers for bioimaging, drug discovery, diagnostics, and therapy (Probst et al., 2013; Bilan et al., 2016; Yao et al., 2018). The main QDs involved in biomedicine contain Cd-based QDs (e.g., CdSe, CdTe, CdS) or Cd-free QDs (e.g., InP, $\mathrm{CuInS}_{2}, \mathrm{AgInS}_{2}, \mathrm{Ag}_{2} \mathrm{~S}, \mathrm{WS}_{2}, \mathrm{ZnO}$, silicon, GQDs, $\mathrm{CuS}$ ) ( $\mathrm{Xu}$ et al., 2016; Mo et al., 2017). However, the potential hazards of QDs have become a critical issue that must be further addressed prior to clinical use.

\section{Organic Nanomaterials}

Optical nanoagents (e.g., QDs, UCNPs, metal nanoclusters, carbon, and silica-based nanomaterials) have been widely employed for molecular imaging. However, inorganic NPs usually suffer from critical safety concerns, including heavy metal poisoning, and not being susceptible to fast clearance from the body. To facilitate clinical translation, organic systems are generally preferred.

Fluorogens with aggregation-induced emission (AIEgens), the typical organic nanomaterials, exhibit very weak emission in the molecular state but high fluorescence in the aggregated state (Luo et al., 2001). They are usually arranged in a rotorlike conformation. In the free state, intermolecular collisions cause the consumption of energy in the form of non-radiative transition, while in the aggregated state, the restriction of intramolecular motion (RIM) ensures the release of exciton energy via a radiative pathway, intensifying the emission (Mei et al., 2015). Through intelligent modulation, AIEgens can emit a broad wavelength in the ultra-violet (UV) to NIR region, accompanied with certain attractive qualities. For example, they enhance the chance of intersystem crossing, thereby increasing the production of free radicals for PDT (Hong et al., 2009; $\mathrm{Xu}$ et al., 2015) and strengthen the conversion of non-radiative relaxation to generate thermal energy for PA imaging and PTT (Mei et al., 2014; Geng et al., 2015). Also, some biomolecules (e.g., target or chelation agents) can be integrated into AIEgens without affecting their AIE properties (Hong et al., 2011; Hu et al., 2014), which endows them with multiple performances for cancer therapy (Yuan et al., 2015). AIEgens have been successfully combined with PSs, drugs, and several imaging methods to realize efficient theranostic platforms (Chang et al., 2012; Xue et al., 2014). Most AIE systems with multiple functions (or modalities) are composed of several components through an all-in-one approach, suffering from defects in their complex molecular structure and multistep synthesis.

Owing to poor photostability of traditional NIR organic dyes, organic semiconducting agents, including semiconducting polymer nanoparticles (SPNs), and semiconducting molecule nanoparticles (SMNs), have emerged as excellent candidates (Lyu et al., 2016; Pu et al., 2016; Zhu et al., 2016). These agents possess higher absorption coefficients, more tunable optical properties, and controllable dimensions compared with inorganic agents (Hong et al., 2012a; Pu et al., 2014a). The wavelength range 650$1,000 \mathrm{~nm}$ of NIR fluorescence imaging is known as the first NIR window (NIR-I), which can achieve a tissue penetration depth of up to $\approx 1 \mathrm{~cm}$ (Shanmugam et al., 2014). However, when NIRI light passes through or interacts with the tissue, it still suffers from light scattering, tissue absorption, and autofluorescence (Ntziachristos, 2010), leading to a relatively poor signal-to-noise ratio (SNR), and thus is less ideal for deep-tissue imaging. To enhance the imaging depth, the second near-infrared window (NIR-II, 1,000-1,700 nm), a more ideal region with deeper tissuepenetration depth and fewer light-tissue interactions, has been explored recently (Miao and Pu, 2018; Cai et al., 2019). To date, organic semiconducting agents have been utilized for deep-tissue imaging, including NIR-II fluorescence, self-luminescence, and PA imaging, in biomedical fields such as cell (Wu et al., 2010; Pu et al., 2014b), tumor (Wu et al., 2011b), acute edema (Pu et al., 2014b), and cardiovascular imaging (Hong et al., 2014) and in real-time evaluation of drug-toxicity (Shuhendler et al., 2014). However, they also face the major issues of high accumulation in the liver and slow removal from the body due to their large dimensions. Therefore, new designs are needed to increase their 
biodegradability or reduce their sizes so that they are smaller than the renal filtration threshold $(\sim 5 \mathrm{~nm})$ for quick clearance via urine excretion.

Polymer nanoparticles (PNPs) have been widely developed for cancer diagnosis and therapy due to their high extinction coefficients, extraordinary fluorescence intensity, good photostability, biocompatibility and biodegradability, and simple encapsulation of anticancer drugs and/or imaging probes (Pecher and Mecking, 2010; Yang et al., 2012; Feng et al., 2017). Although PNPs can accumulate in the tumor region via the enhanced permeability and retention (EPR) effect (Luk and Zhang, 2014; Tang et al., 2016), cancer-targeting moieties (e.g., proteins, peptides, and aptamers) must be modified onto the NP surface by chemical or physical interaction, resulting in functionalized PNPs with enhanced selectivity and sensitivity for cancer diagnosis and therapy (Liu et al., 2015c; Li et al., 2016; Li and Yang, 2017; Lin et al., 2017). Recently, functionalized PNPs have been designed according to specific cellular processes and disease environments and applied to selectively deliver drugs to the target regions (Vaidya et al., 2011; Krasia-Christoforou and Georgiou, 2013; Herranz-Blanco et al., 2016; Kumar et al., 2018; Wang et al., 2019). Further in vivo work will be required to comprehensively investigate the safety and efficacy of these novel theranostic platforms before clinical application.

\section{OPTICALLY ACTIVE NANOMEDICINE FOR BIOIMAGING}

\section{Fluorescence Imaging}

Fluorescence imaging, as a means to visualize specific organelles within cells or animals, has become a powerful tool for biological research and even for the emerging field of fluorescenceguided surgery. Additionally, fluorescence imaging can capture specific molecular information on the tumor microenvironment (Wolfbeis, 2015).

Among the commonly utilized non-viral vectors, gold nanocages (AuNCs) were investigated for microRNA (miRNA) delivery due to their biological inertness and unique physicochemical features (Skrabalak et al., 2008). Their strong and easily adjustable absorption and scattering in the NIR region demonstrate their feasibility in theranostic uses (Xia et al., 2011). The sizes, shapes or surface chemistry of NPs can mediate their cellular uptakes, macrophage clearance, and biodistribution (Huang and El-Sayed, 2010). NPs with a size of 10-100 nm are more appropriate for cancer diagnosis and therapy since they can escape from renal elimination and accumulate in tumors. Besides size, surface chemistry is another crucial factor for the cellular uptake and biodistribution of NPs in vivo (Chen et al., 2005b). Bao et al. (2017) have successfully fabricated a series of differently-sized miRNA delivery systems, miR-26a-loaded, hyaluronic acid (HA)-modified, polyetherimide-conjugated PEGylated AuNC ternary nanocomplexes (PPHAuNCs-TNCs), and monitored them through fluorescence and PA imaging. First, they prepared PEGylated AuNCs (PAuNCs) by employing HS-PEG-OMe (MW 5000) to replace the PVP layer through an $\mathrm{Au}-\mathrm{S}$ bond to extend the circulation period of the NPs in the blood and enhance their stability. Secondly, polyethyleneimine (PEI)-conjugated PAuNCs (PPAuNCs) were obtained by conjugation of PEI onto the surface of the PAuNCs to condense miRNAs, utilizing 11-mercaptoundecanoic acid (MUA) as a linker. Thirdly, miRNAs were encapsulated with differently-sized PPAuNCs via electrostatic interaction to obtain miRNA-loaded, PEI-conjugated PEGylated AuNC binary nanocomplexes (PPAuNCs-BNCs). Finally, to neutralize their slightly positive charge and enhance their stability and biocompatibility, a small amount of HA was added to PPAuNCs-BNCs to obtain the final product, PPHAuNC-TNC (Figure 1A).

miRNAs were labeled with Cy5.5, a NIR fluorescent dye, so as to visualize the effect of NP size on biodistribution in vivo. Figure $1 \mathrm{~B}$ shows real-time images of differently-sized PPHAuNCs-TNCs in BEL-7402 tumor-bearing nude mice. Notably, the fast tumor accumulation of PPHAuNCs-30-TNCs was clearly observed at $0.5 \mathrm{~h}$ and approached saturation at $6 \mathrm{~h}$ post-injection. For the PPHAuNCs-30-TNC group, sustainable maintenance of the relatively high fluorescence density was found at the tumor site at $24 \mathrm{~h}$, which was similar to the PPHAuNCs50-TNC group. However, slower and less tumor accumulation was observed for the PPHAuNCs-50-TNC group than for the PPHAuNCs-30-TNC group. In addition, no obvious fluorescence signals were detected at the tumor site $24 \mathrm{~h}$ after injection in the PPHAuNCs-70-TNC and naked Cy5.5-labeled miRNA groups. This was likely due to the accumulation at the tumor site being too weak to be examined or its rapid clearance from the body. The different biodistribution profiles of the three NPs clearly indicated that NP accumulation in the tumor could be ascribed to not only the EPR effect but also diffusion, which largely relied upon the morphology, dimensions, and surface charges of the NPs and the physicochemical properties of the interstitial matrix. The above results confirmed that the PPHAuNCs-30TNCs and PPHAuNCs-50-TNCs efficiently delivered miRNAs into the tumor.

Abraxane is the trade name of paclitaxel (PTX)-loaded human serum albumin (HSA) NPs, which is an example of clinical success of nanomedicine against cancer (Ma and Mumper, 2013). HSA is usually applied to encapsulate certain theraputic drugs to promote their biocompatibility (Desai, 2016). Wang et al. (2016a) constructed a targeted agent by employing PTX and AIEgens for cancer imaging and therapy. The theranostic nanoplatform included four elements: (1) AIEgens conjugated with HSA for imaging; (2) cyclic arginine-glycine-aspartic acid (cRGD)-modified HSA for specific recognition; (3) HSAfunctionalized polypyrrole (PPy) for thermotherapy; (4) PTX as a chemotherapy agent and mediating the protein assembly. Firstly, HSA-AIEgens was synthesized by introducing AIEgens into a hydrophobic pocket of HSA, which endowed AIEgens with strong fluorescence. Notably, due to the superb features of AIEgens, the final product exhibited strong fluorescence intensity even after conjugation with quencher-PPy. Next, the imaging performance of the AIEgens-based nanoplatform was investigated to assess tumor treatment efficacy in mice. With intravenous injection for about $1 \mathrm{~h}$, AIEgens fluorescences were observed in the whole bodies of the mice, which were associated with the large amounts of NPs in blood. Thereafter, 
A

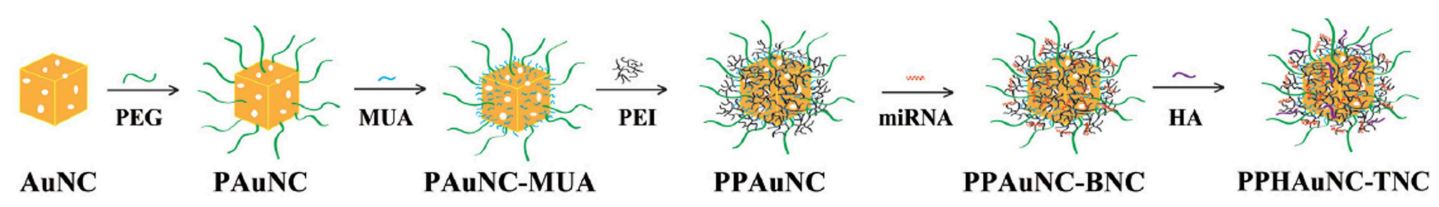

\section{$\begin{array}{lllll}0.5 h & 1 h & 6 h & 12 h & 24 h\end{array}$}

B
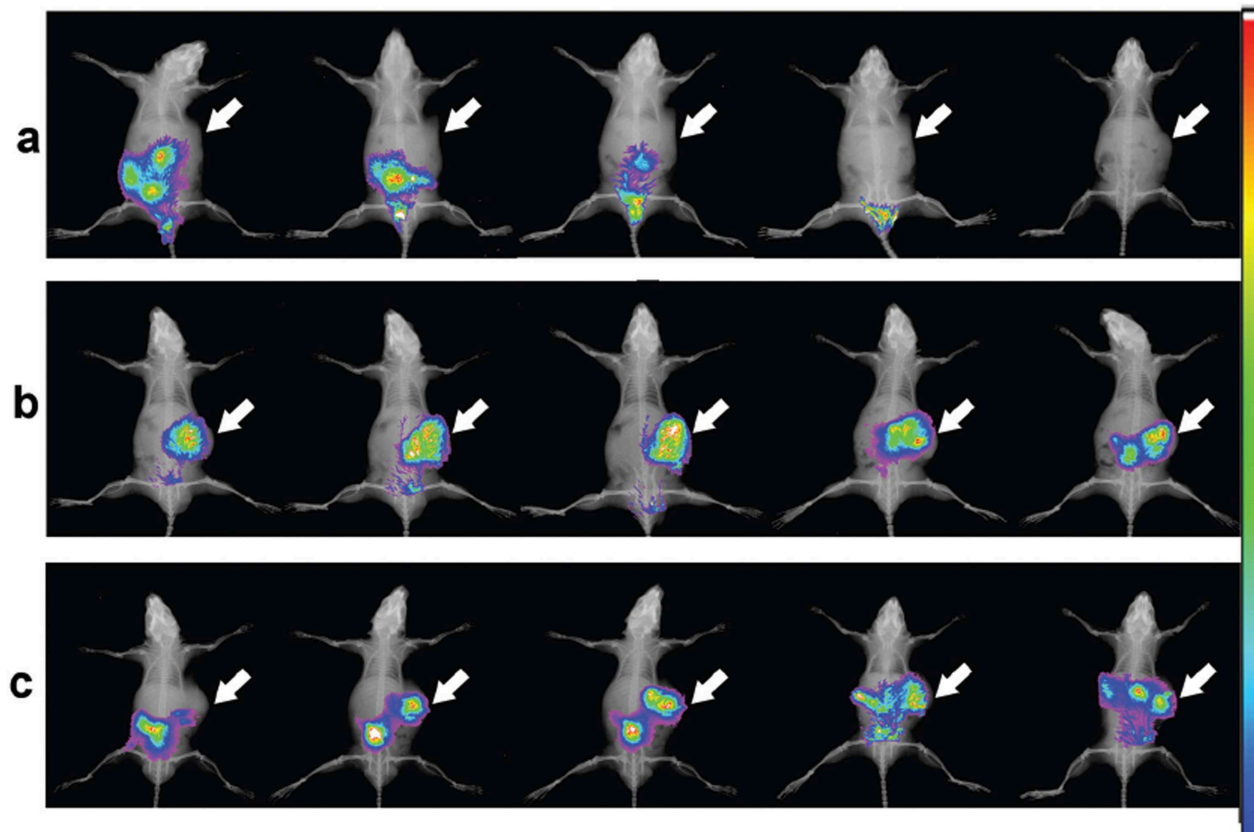

$-1.8 e+003$

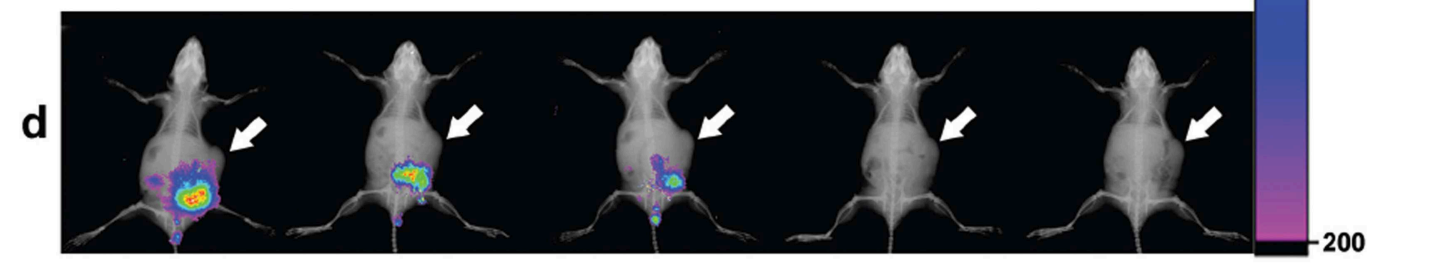

FIGURE 1 | (A) Formation of PPHAuNC-TNC. (B) in vivo biodistribution of differently-sized PPHAuNCs-TNCs. After intravenous injection with (a) naked Cy5.5-miRNAs (control group), (b) PPHAuNCs-30-TNCs, (c) PPHAuNCs-50-TNCs, and (d) PPHAuNCs-70-TNCs.

the nanoagent showed elevated accumulation in tumors with enhanced fluorescence vs. time, which verified its ability to target tumors specifically. Also, the fluorescence of the nanoagent in mice still remained strong after injection for $48 \mathrm{~h}$, which indicated the superior performance of AIEgens in bioimaging and their superb long-lasting retention in vivo.

\section{Persistent Luminescence Imaging}

Persistent luminescence is the phenomenon whereby luminescence lasts for several seconds to even days after switching off the excitation source. Generally, two types of active centers, i.e., traps and emitters, contribute to the generation of persistent luminescence. Intrinsic lattice defects or codopants in the host material act as the traps, which are just a few electron volts $(\mathrm{eV})$ below the conduction bands. The emitters are usually lanthanide or transition metal ions. The emergence of persistent luminescence covers four consecutive processes: the formation of charge carriers, trapping of charge carriers, release of the trapped charge carriers, and recombination of the released charge carriers to generate emission.

$\mathrm{ZnGa}_{2} \mathrm{O}_{4}: \mathrm{Cr}^{3+}$ is gaining considerable attention because of its strong NIR persistent luminescence upon UV excitation (Bessiere et al., 2011). Maldiney et al. (2014a) introduced the new generation of $\mathrm{ZnGa}_{2} \mathrm{O}_{4}: \mathrm{Cr}^{3+}$ nanoprobes whose persistent luminescence could be directly charged in vivo by incident light with deep penetration and low energy. Low-temperature sintering was adopted to prepare the nanoprobes. The excitation spectrum of n-ZGO photoluminescence showed a broad spectral range from ultraviolet to red light containing four bands (Figure 2A, solid black line). The bands at 245, 290, and 425-560 $\mathrm{nm}$ corresponded to an exciton energy higher than the bandgap of $\mathrm{ZnGa}_{2} \mathrm{O}_{4}$, interband excitation, and $\mathrm{Cr}^{3+} \mathrm{d}$ $\mathrm{d}$ transitions, respectively. The ${ }^{4} A_{2} \rightarrow{ }^{4} T_{2}$ absorption band was partly ascribed to weaker absorption of tissue domains 


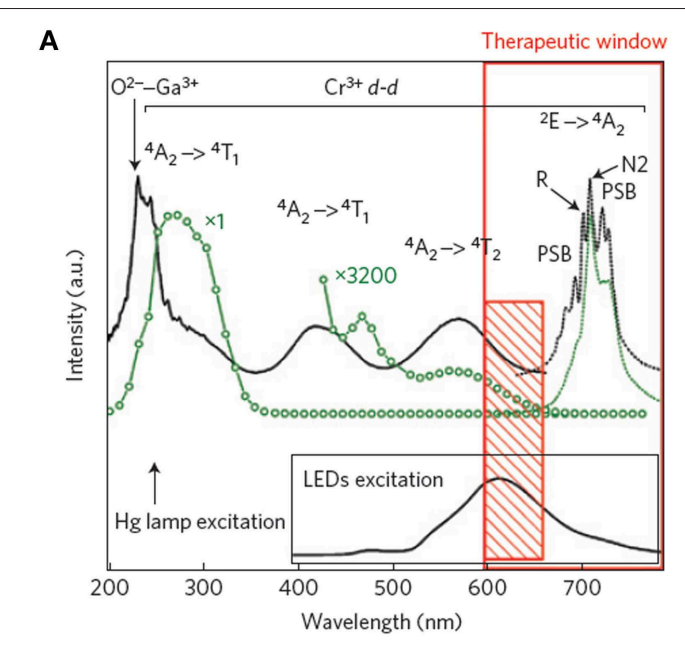

C

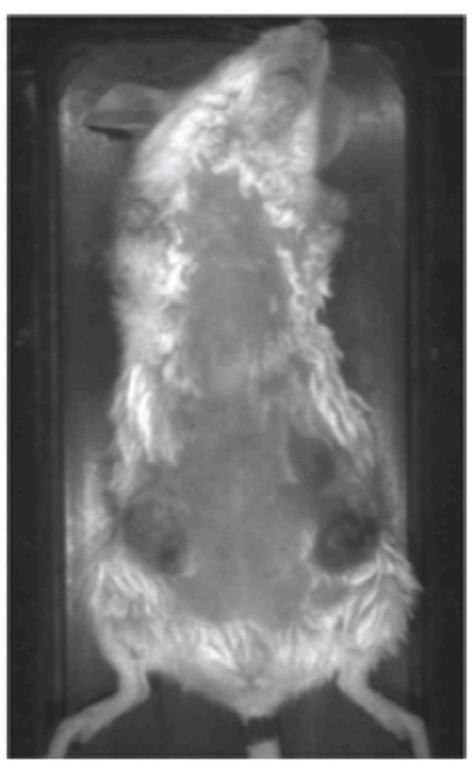

B

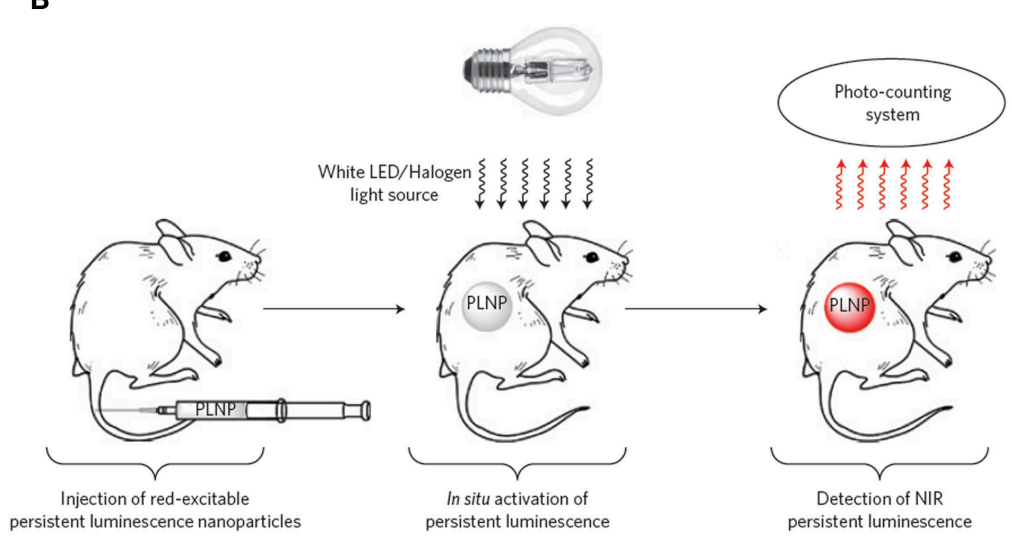

D

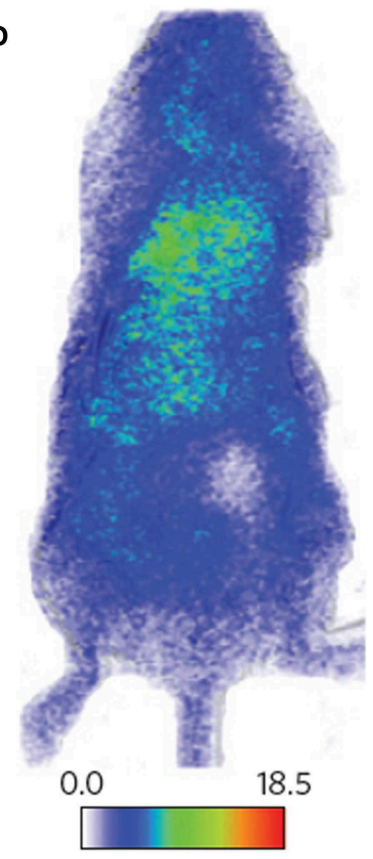

E

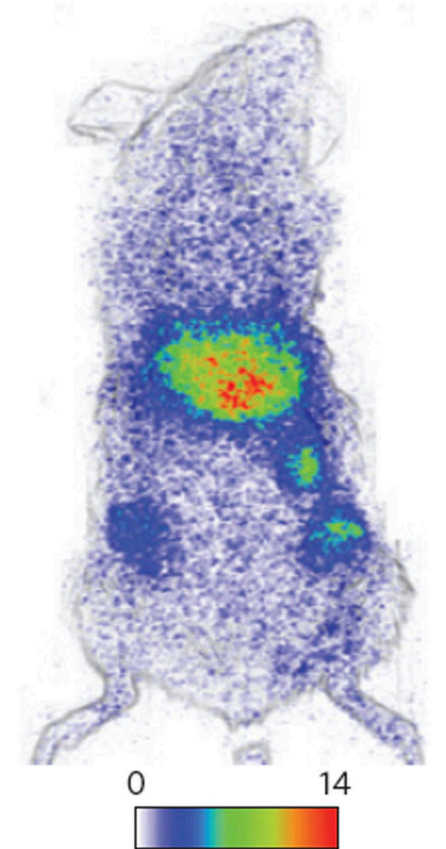

FIGURE 2 | (A) Photoluminescence excitation (black solid line) and emission (black dotted line) spectra of $\mathrm{Cr}^{3+}$ doped n-ZGO. (B) Schematic representation of in vivo imaging after in situ activation of persistent luminescence nanoparticles (PLNPS). (C) Optical image of a tumor-bearing mouse. (D) Persistent luminescence image of a tumor-bearing mouse $2 \mathrm{~h}$ after injection of ZGO-PEG NPS. (E) Persistent luminescence image of a tumor-bearing mouse immediately after LED illumination, $4 \mathrm{~h}$ after injection of ZGO-PEG NPs.

(red rectangle, Figure 2A). The n-ZGO PL emission (Figure 2A, dotted black line) consisted of the ${ }^{2} E \rightarrow{ }^{4} A_{2}$ bandgap of $\mathrm{Cr}^{3+}$. The mechanism for $\mathrm{ZnGa}_{2} \mathrm{O}_{4}: \mathrm{Cr}^{3+}$ persistent luminescence under visible light was related to antisite defects around the $\mathrm{Cr}^{3+}$ ion. Then, $\mathrm{ZnGa}_{2} \mathrm{O}_{4}: \mathrm{Cr}^{3+}$ nanoprobes were directly injected into mice without preliminary activation (Figure 2B). In the first $2 \mathrm{~h}$, there was no difference in the biodistribution between tumor-bearing and healthy mice (Figures 2C,D). However, persistent luminescence images of tumor-bearing mice clearly showed the tumors $4 \mathrm{~h}$ after PEGylated ZGO administration and following visible activation using the orange/red LED source (Figure 2E).
Unfortunately, the persistent luminescence peak (around $700 \mathrm{~nm}$ ) of $\mathrm{ZnGa}_{2} \mathrm{O}_{4}: \mathrm{Cr}^{3+}$ made it difficult to use for deep tissue imaging. Also, the emissions of Near-infrared persistent luminescence NPs (NPLNPs) quickly decays over time, leading to lower SNRs. Therefore, superior nanoprobes with higher SNRs are urgently needed for bioimaging. Shi et al. (2018) fabricated a desirable NPLNP, $\mathrm{mSiO}_{2} @ \mathrm{Gd}_{3}-\mathrm{Ga}_{5} \mathrm{O}_{12}: \mathrm{Cr}^{3+}, \mathrm{Nd}^{3+}$ $\left(\mathrm{mSiO}_{2} @ \mathrm{GGO}\right)$, for bioimaging and cancer treatment. The $\mathrm{mSiO}_{2} @ \mathrm{GGO}$ NPs showed an intense emission at $745 \mathrm{~nm}$ using 254-nm excitation. The NIR emission was ascribed to the $\mathrm{Cr}^{3+}$ spin-allowed ${ }^{4} \mathrm{~T}_{2} /{ }^{4} \mathrm{~A}_{2}$ transition. The 258-nm excitation band was attributed to the GGO host absorption. After the 
nanoprobes were injected into mice, their luminescence signals were detected throughout the body in $5 \mathrm{~min}$. An intensive luminescence was observed in the liver due to nanoprobes captured by the reticuloendothelial system (RES). The signal intensities of the nanoprobes were even still maintained in the liver after $60 \mathrm{~min}$. This suggested that the nanoprobes could achieve long-duration imaging in vivo using their NIRI luminescence. Also, the $\mathrm{mSiO}_{2} @ G G O$ NPs could realize 2$\mathrm{cm}$ tissue imaging with high SNR $(\approx 5.5)$. Three main emission peaks were detected at $888,1,067$, and $1,338 \mathrm{~nm}$, corresponding to the eletronic transitions of $\mathrm{Nd}^{3+}$ from the excitation level ${ }^{4} \mathrm{~F}_{3 / 2}$ to the bottom states ${ }^{4} \mathrm{I}_{9 / 2},{ }^{4} \mathrm{I}_{11 / 2}$, and ${ }^{4} \mathrm{I}_{13 / 2}$ with 808 $\mathrm{nm}$ irradiation, respectively. Among them, the emission peak at $1067 \mathrm{~nm}$ was predominant, demonstrating high NIR-II emission efficiency. After the nanoprobes were subcutaneously injected into the abdomens of mice, a remarkable NIR-II luminescence signal was detected at the injection site, while negligible intensity was determined at other abdomen areas, suggesting that the nanoprobes can be applied for in vivo imaging in the NIRII region.

PLNPs have gained more attention in deep-tissue bioimaging due to their emission in the NIR region, lack of in-situ excitation, and high SNR (de Chermont et al., 2007; Abdukayum et al., 2013). In particular, $\mathrm{Cr}^{3+}$-doped PLNPs can produce renewable persistent luminescence under tissue-penetrating LED light, which indicates that the imaging performance of PLNPs is no longer restricted by the light-emitting lifetime (Li et al., 2015a). Chen et al. (2016b) fabricated a novel luminescence probe for bioimaging by combining PLNPs and metallic sulfide. The nanoprobe is attractive due to its ultrasensitive switchon imaging response to targeted recognition. The PLNP, $\mathrm{Zn}_{1.1} \mathrm{Ga}_{1.8} \mathrm{Ge}_{0.1} \mathrm{O}_{4}: \mathrm{Cr}^{3+}$, served as the light source due to its renewable persistent luminescence. CuS NP is not only as a PTT agent but also serves as a quenching agent for photothermal conversion and NIR absorption. A peptide catalyzed by matrix metalloproteinase (MMP) was introduced to construct the nanoprobe. Further modification with mercaptoPEG and polypeptide endows the nanoprobe with desirable biocompatibility and tumor specificity. To evaluate the activation performance of the nanoprobe, it was intravenously injected into mice after being treated by UV light for $10 \mathrm{~min}$. Remarkable luminescence was clearly detected at the tumor site of mice for about $2 \mathrm{~h}$, showing efficient MMP activation and the superior tumor-targeting recognition of the probe.

Some PLNPs consisting of host nanomaterials and codopants have been extensively investigated; please see details in Table 1 (Liu et al., 2019a).

\section{Near-Infrared Surface-Enhanced Raman Scattering (NIR SERS) Imaging}

NIR optical nanoprobes have received increasing attention as multimodal therapeutic reagents for bioimaging and photothermal tumor elimination. For optical imaging, SERS has emerged as an attractive method with intense signal response and good specificity (Gandra and Singamaneni, 2013; Zhang et al., 2018). A narrow bandwidth, high resistance to photobleaching, and autofluorescence make SERS an effective tool for non-invasive single-cell assay, rapid disease diagnosis, and nanomedical imaging (Maiti et al., 2012; Guerrini et al., 2017). Three parts, including a noble metal matrix, Raman active reporters, and a biocompatible surface coating, constitute a typical NIR SERS nanoprobe.

The NIR SERS probe is usually prepared through direct modification of a reporter molecule (e.g., a dye) onto the metal surface. However, the electrostatic force-based metaldye framework leads to structural instability, particularly in physiological environments (Wang et al., 2013b). Strong cytotoxicity is another defect that limits the uses of dye-based SERS probes in nanomedicine. Outer covering with hydrophilic thiolated polyethylene glycol (PEG-SH) can evidently improve the biocompatibility of the metal-dye system. However, competitive binding of polymer and dye reporter with the noble metal can reduce the SERS signal intensity of probes. Also, this kind of PEG-based modification is unsuitable for PTT owing to its labile steric conformation, which is subject to morphological change (melting) upon high-power NIR irradiation (Wang et al., 2012). Liu et al. (2015d) developed environmentally friendly NIR SERS nanoprobes instead of using highly toxic organic dyes. Multifunctional conducting polymer (CP), acting as an outer modification layer and Raman-active molecule, was immobilized on the surface of gold nanorods (GNRs). The GNR-CP nanostructure was separately fabricated through oxidatively chemical polymerization, utilizing two different combinations of monomer and oxidant, namely pyrrole/ $/ \mathrm{FeCl}_{3}$ and aniline/ $\left(\mathrm{NH}_{4}\right)_{2} \mathrm{~S}_{2} \mathrm{O}_{8}$. Rod-shaped GNRs were synthesized with average dimensions of $42.3 \pm 6.9 \mathrm{~nm}$ long and $9.6 \pm$ $1.4 \mathrm{~nm}$ wide (Figure 3A). Such nanoparticles are considered to have an EPR effect and long-term retention in tumors. The UV-vis-NIR absorption spectra of GNRs showed a transverse and a longitudinal SPR peak at around 515 and $800 \mathrm{~nm}$, respectively. Figure $3 \mathrm{Ab}, \mathrm{c}$ show different constructions of two probes, GNR-polyaniline(PANI) and GNR-polypyrrole(PPy). Remarkable shifts toward longer wavelengths and reductions in absorbances were observed in the absorption curves of GNR-CPs (Figure 3Ad). The SERS fingerprints of the nanoprobes were dependent on excitation light source. Negligible SERS signal were detected as the probes were illuminated with $514.5 \mathrm{~nm}$ light. In comparison, very strong SERS signals appeared upon $785 \mathrm{~nm}$ excitation. The strongest peaks were located at $945 \mathrm{~cm}^{-1}$ for GNR-PPy and $1,170 \mathrm{~cm}^{-1}$ for GNR-PANI (Figure 3Ae,f), which were ascribed to in-plane distortion and bending vibrations of $\mathrm{C}-\mathrm{H}$ in the quinoid group, respectively.

A549 cells exposed with the probe were investigated by utilizing the above NIR SERS imaging. After cell exposure to GNRs, the SERS images exhibited a very weak response from cellular elements in the $600-1,700 \mathrm{~cm}^{-1}$ range (Figure $3 \mathrm{Ba}-\mathrm{c}$ ). However, distinct SERS signals were obtained from cells exposed to GNR-PANI (Figure 3Be-g) or GNR-Ppy (Figure 3Bh-j). The results demonstrated that the probes were mainly distributed in the cytoplasm. The characteristic signals from the cytoplasm were detected clearly in the SERS spectra (Figure 3Bd), displaying the availability of SERS probes in the sophisticated cellular environment. Moreover, cancer cells were cotreated with the two 
TABLE 1 | Persistent luminescence NPs for bio-imaging and therapy.

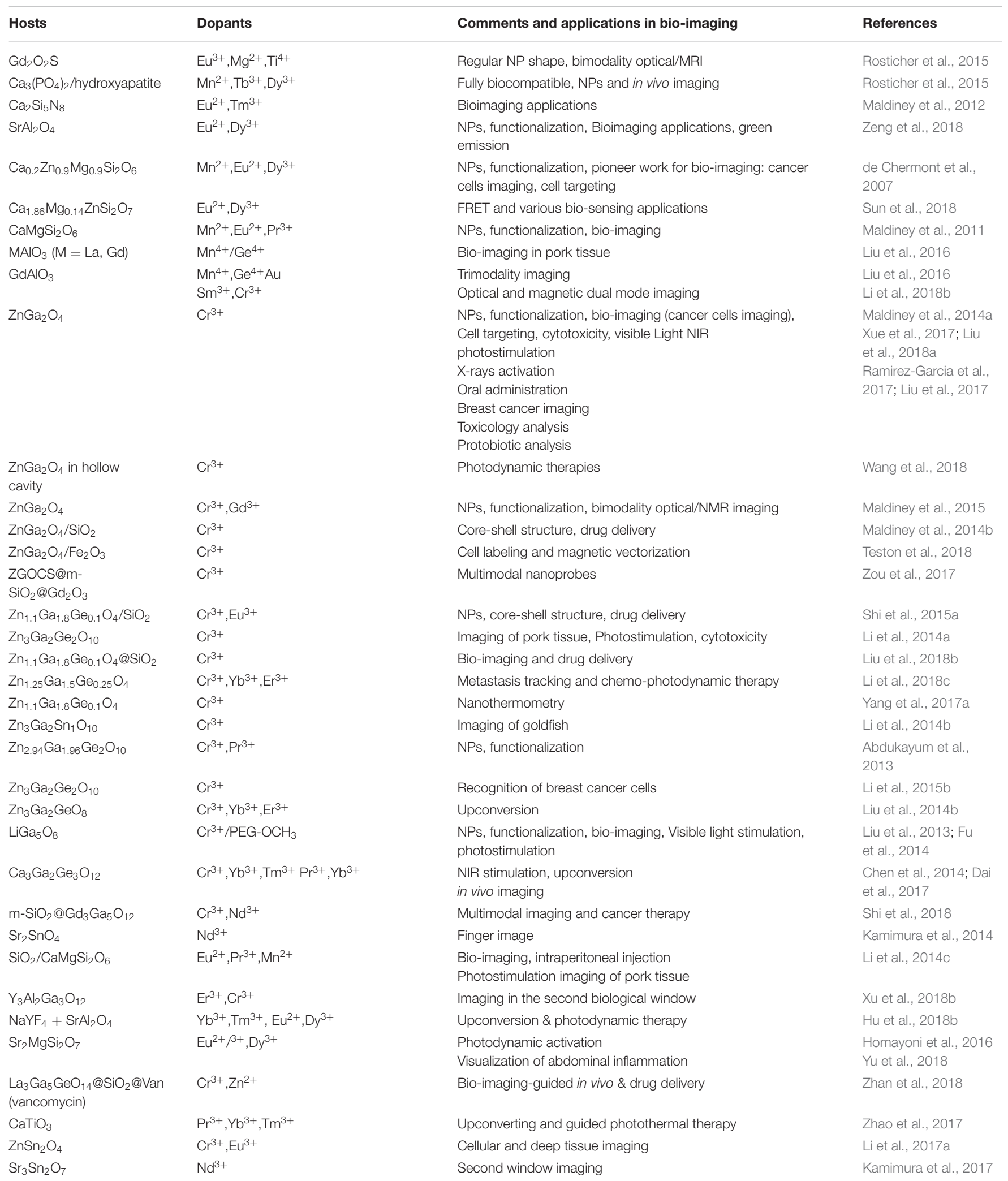



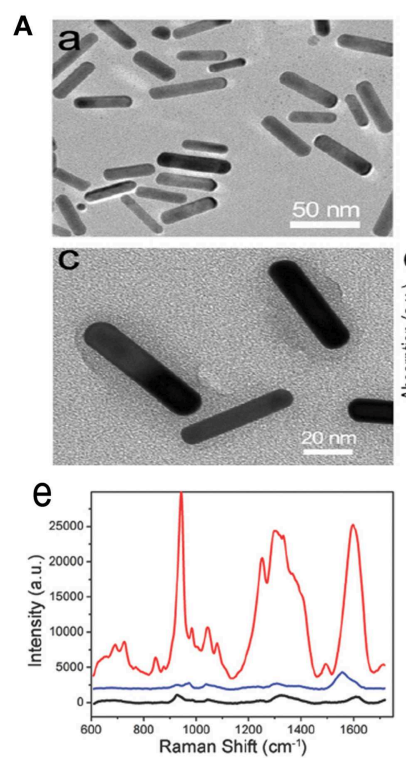
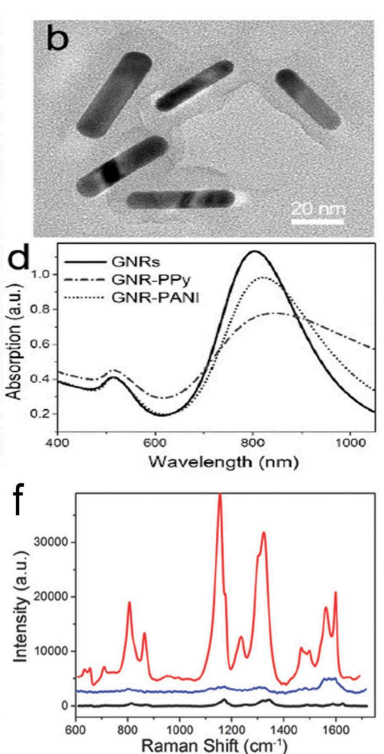

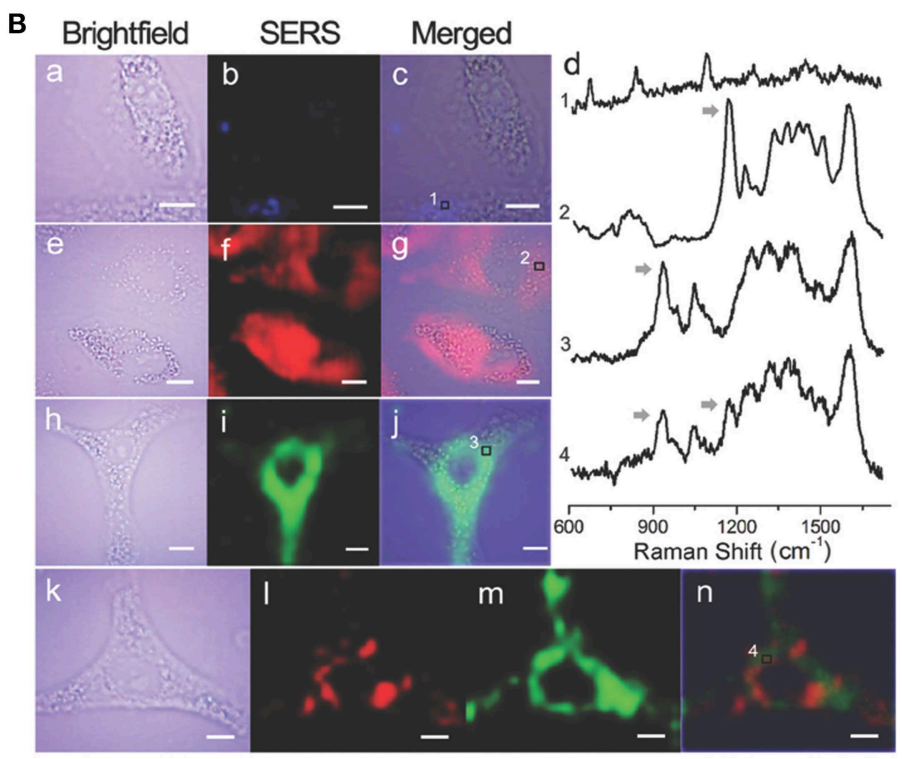

FIGURE 3 | (A) Characterization of SERS nanoprobes: (a-c) TEM images of GNRs, GNR-PANI, and GNR-PPy, respectively. (d) UV-vis-NIR absorbance spectra of the nanostructures. Raman spectra of GNR-PPy (e) and GNR-PANI (f) under $785 \mathrm{~nm}$ (red line) and $514.5 \mathrm{~nm}$ (blue line) laser excitation, respectively. Black lines show the normal Raman spectra of CP molecules. (B) NIR SERS imaging of A549 cells after incubation with GNRs (a-c), GNR-PANI (e-g), and GNR-PPy (h-j) for 4 h. (d) Raman spectra in the cytoplasmic compartments. Arrows indicate the Raman peaks used for SERS imaging. (k-n) Two-color imaging after incubation with a mixture of two tags [brightfield image (k), GNR-PANI (I), GNR-PPy (m), merged image (n)].

probes. Figure 3Bl,m show SERS images of the probes and the individual response distribution in cells. Notably, each mapping was separate, which was attributed to the narrow bandwidth of the Raman peaks.

Silver bumpy nanoshell (AgNS) has been explored as a highly active NIR SERS nanoprobe for the biomedical field (Premasiri et al., 2018). AgNS has intense scattering in the NIR-region, where its SERS enhancement factor is higher than those of gold nanorods or nanospheres. Also, AgNS has strong NIR absorption, which is useful for PA imaging. Additionally, AgNS do not induce any in vivo toxicity, as confirmed by cytotoxicity tests (Baumberg et al., 2017). Cha et al. (2017) demonstrated dual-modal detection of sentinel lymph nodes (SLNs) using a silica-coated silver bumpy nanoshell probe $\left(\mathrm{AgNS} @ \mathrm{SiO}_{2}\right)$ : imaging the SLN with a PA signal and in vivo multiplex identification of targets in the located region with SERS. Using the correctional Stöber approach, the surface of AgNS was covered by silica-shell and finally treated with bovine serum albumin (BSA) to enhance biocompatibility. AgNS@SiO possesses some advantages, e.g., high dispersion stability, facile surface modification, and good biocompatibility. PA imaging was adopted to detect the accumulated regions of $\mathrm{AgNS} @ \mathrm{SiO}_{2}$, and the SERS signals were analyzed to identify different ratios of several kinds of Raman-labeled AgNS@SiO 2 . After three different types of Raman-labeled AgNS@SiO 2 probes were injected into rat's left front paw pad, respectively, their PA and SERS signals were monitored and analyzed to locate the SLN and identify the targeted AgNS@SiO 2 . They successfully obtained PA images and SERS spectra using the portable-Raman system with a 785-nm NIR-excited laser. This dual-modal imaging system may be utilized in diagnostic fields, e.g., multiplex cancer marker detection in vivo.

\section{Photoacoustic (PA) Imaging}

Photoacoustic (PA) imaging, a new method of visualization via photoacoustic response, has increasing potential in nanomedical fields (Kim et al., 2010). PA imaging has been explored to visualize biostructures from organelles to cells to organs (Wang and $\mathrm{Hu}, 2012$ ). A contrast agent absorbs the excitation energy and converts it into thermal energy. Thus, a wideband ultrasound emission is produced due to heat-induced transient thermoelastic expansion, which is collected with an acoustic detector and transformed into PA images. PA imaging combines the good specificity of optics and the deep-tissue transmission of ultrasound (US), which overcomes the restrictions of traditional imaging (Nie and Chen, 2014).

Perylene-3,4,9,10-tetracarboxylic diimide (PDI) and its derivatives have been extensively applied to fabricate diverse electronic devices based on their extraordinary physicochemical properties, easy functionalization, and extremely low price (Perrin and Hudhomme, 2011; Birel, 2017). Fan et al. (2015) constructed an efficient PDI-based NIR-absorptive contrast nanoagent enveloped by micelle for PA imaging of deep brain tumor in living mice. To make PDI absorb in the NIRregion, tertiary amine and diimide as donor and acceptor were introduced to fabricate a classical donor- $\pi$-acceptor system for increasing redshift efficacy. Water-soluble PDI NPs were prepared by wrapping with amphiphilic PEG derivatives (Figure 4A). These PDI NPs displayed strong NIR absorption at around $700 \mathrm{~nm}$ in aqueous solution (Figure 4B). After 2-h 

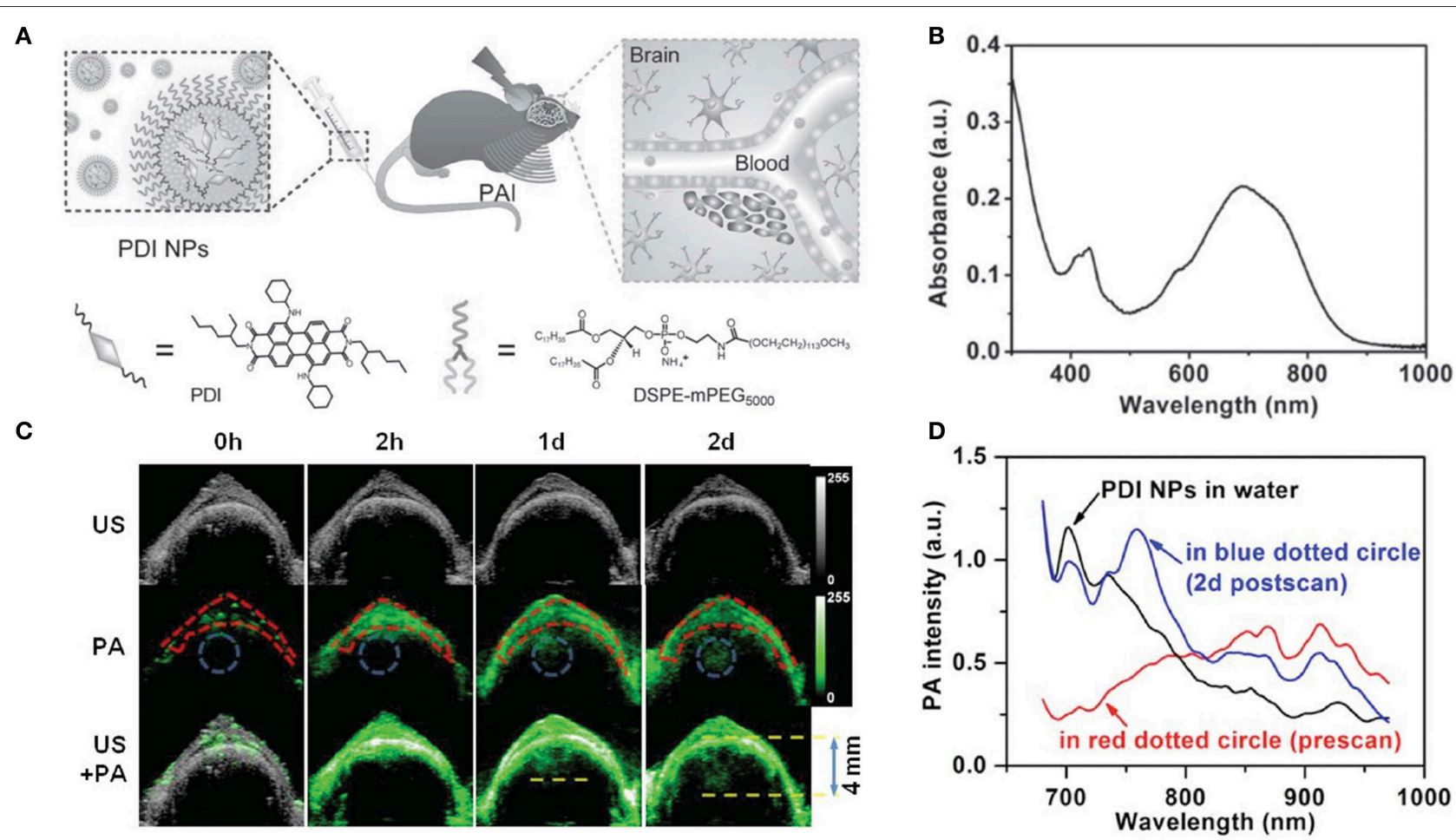

FIGURE 4 | (A) Schematic illustration of the PA imaging process of brain tumor in vivo by PDI NPs. (B) UV-vis-NIR absorption spectrum of PDI NPs in aqueous solution. (C) Ultrasonic (gray), photoacoustic (green), and their overlay brain coronal sections of the tumor model after tail vein injection of PDI NPs. (D) PA spectra of PDI NPs in different media. Skull region in the red dotted circle of (C) before NP injection (red line) and tumor region in the blue dotted circle of (C) after 2 d NP injection (blue line).

injection, the PA signal at the skull region (red dotted circle) intensified greatly due to the circulation of PDI NPs in the blood vessel. After 1-d injection, a significant PA signal appeared in the brain tumor region (blue dotted circle) of mice, but no signal was found in the control group. Also, a 4.0-mm depth of the tumor region was more clearly observed after 2 - $\mathrm{d}$ injection compared with a shallower depth $(\sim 3.0 \mathrm{~mm})$ after 1 -d injection (Figure 4C), which was attributed to the enhanced tumor penetration depth of NPs with time. The biodistribution of PDI NPs in brain tumor was verified by measuring PA spectra. Strong PA intensities at 700 and $735 \mathrm{~nm}$ (blue line) were clearly observed in the tumor region after 2-d NP injection (Figure 4D), which was consistent with the NIR absorption peak of PDI NPs (Figure 4B). This indicated the successful accumulation of PDI NPs in the tumor.

Carbon dots (CDs) are promising carbon-based imaging probes due to their excellent biocompatibility, water solubility, and photostability (Yang et al., 2009; Li et al., 2012, 2013). However, the main challenge of CDs for practical application is guaranteeing their biodegradability (Tang et al., 2013; Ge et al., 2015; Jiang et al., 2015). Moreover, the mechanisms of the interaction between CDs and low-energy photons are being investigated to obtain high resolution and contrast for CD optical imaging. Lee et al. (2016) synthesized a biocompatible and $\mathrm{N}$-doped type of $\mathrm{CD}(\mathrm{N}-\mathrm{CDs})$ for $\mathrm{PA}$ imaging. $\mathrm{N}$-CDs were prepared by solvothermal carbonation using citric acid and $\mathrm{HNO}_{3}$ as a $\mathrm{C}$ and $\mathrm{N}$ source. $\mathrm{N}$-CDs showed strong absorption in the NIR region $(680-800 \mathrm{~nm})$. N-CDs with high contents of $\mathrm{N}$ atoms raised the ambient temperature more quickly upon NIR (680-808 nm) laser irradiation. This was because a high $\mathrm{N}$ content produced more bandgaps so that the carrier could be captured from LUMO to a bandgap and relaxed between bandgaps. N-CDs exhibited higher heat conversion efficiency than conventional PA contrast agents (e.g., GNR and methylene blue) under the same optical density. Therefore, N-CDs could produce sufficiently intense PA effects to realize non-invasive imaging and thermal therapy in vivo. The researchers carried out time-resolved PA imaging of SLNs of Sprague-Dawley rats and evaluated the biodegradability of N-CDs through renal clearance. The relative PA signal was suddenly enhanced in SLNs 30 min after hypodermic injection of N-CDs and reduced gradually until $180 \mathrm{~min}$. Meanwhile, the PA signal from the bladder area rose intensively at $100 \mathrm{~min}$, suggesting the effective removal of $\mathrm{N}$-CDs to the urine. For tumor PTT, the NCDs displayed complete tumor ablation without recurrence for $\mathrm{Balb} / \mathrm{c}$ nude xenograft HepG2 tumor model mice with a tumor volume of $27 \mathrm{~mm}^{3}$ upon 808-nm NIR laser irradiation. NCDs, as a kind of NIR-absorbing nanomaterial, will become a potent contrast agent for PA bioimaging and for improving PTT efficacy. 
A new type of optical nanoagent, semiconducting polymer NP (SPNP), provides an inspiring strategy for solving the nanotoxicity problem ( $\mathrm{Li}$ and $\mathrm{Pu}, 2019$ ). SPNPs with highly conjugated structures exhibit excellent photothermal conversion efficiency and photostability (Li et al., 2018d). Moreover, the molecular versatility of SPNPs is not only beneficial to facile tuning of the spectrum independent of size or morphology but also allows the skeleton or side chain to be easily modified for on-demand functionalization (Feng et al., 2013). Based on these merits, SPNPs have been used as promising PA agents for biomarkers and cancer detection (Lyu and $\mathrm{Pu}, 2017$ ). Furthermore, preliminary investigation has revealed that SPNPs have the advantage of being degradable NIR-I nanoagents (Lyu et al., 2018). Nevertheless, whether they can perform as biodegradable NIR-II nanoagents has not been explored. Jiang et al. (2019) fabricated metabolizable NIR-II SPNP-based agents for PA imaging. By introducing the strong electron-withdrawing group benzobisthiadiazole, the synthesized SPNPs (SPNP-PT, SPNP-DT, and SPNP-OT) showed strong absorption at $1,079 \mathrm{~nm}$ and superior photothermal conversion efficiencies at $1,064 \mathrm{~nm}$. Owing to the oxidizable thiophene group and hydrolyzable PEGbased matrix in their skeletons, three NIR-II SPNPs $(\sim 30 \mathrm{~nm})$ could be effectively decomposed into smaller sizes NIR NPs $(\sim 1 \mathrm{~nm})$ by myeloperoxidase or lipase with high abundance in vivo. Further, the SPNPs were completely eliminated in 15 days through renal or hepatobiliary metabolism in living mice. Especially, SPNP-PT demonstrated good SNRs for PA imaging of tumor or brain vasculature in mice under a systematic dosage $\left(2.5 \mathrm{mg} \mathrm{kg}^{-1}\right)$ lower than that of other reported agents $\left(\geq 10 \mathrm{mg} \mathrm{kg}^{-1}\right)$.

Overall, various nanomaterials have been exquisitely designed for deep-tissue PA imaging. PA contrast agents are mainly categorized into inorganic and organic nanoagents based on their structures (Table 2) (Wang et al., 2016b).

\section{OPTICALLY ACTIVE NANOMEDICINE FOR TARGETED THERAPY AND DRUG DELIVERY}

\section{Photodynamic Therapy (PDT)}

Photodynamic therapy (PDT) employs photosensitizers (PSs) and light of a specific wavelength in combination with molecular oxygen to generate reactive oxygen species (ROS) that kill cancer cells through the oxidation of important biomolecules and organelles (Zhou et al., 2016a). The introduction of NPs in PSs provides the following advantages: (1) effective PS delivery to the target site, (2) easy phase transfer of hydrophobic PSs into an amphiphilic bloodstream to increase circulation time, (3) use of the EPR effect for the effective diffusion of PSs into tumors, (4) surface modification with various molecules, enhancing the cellular uptake and targeting, and (5) multiple functionality by combining the properties of NPs (e.g., multimodal imaging) with those of PSs (Lucky et al., 2015). A myriad of inorganic and organic nanostructured PSs, e.g., gold NPs (Dykman and Khlebtsov, 2012), metallic oxides (Bechet et al., 2008), carbon-based materials (Albert and Hsu, 2016), mesoporous silica (Montalti et al., 2014), polymeric micelles (Elsabahy et al., 2015), and UCNPs (Wang et al., 2017), have been developed for image-guided PDT therapies (Lan et al., 2019).

Recently, AIE PS, a new family of PSs, has been gaining more attention ( $\mathrm{Hu}$ et al., 2018a). Owing to the inhibition of non-radiative energy consumption and RIM, AIE PSs exhibit enhanced signal intensity (Alifu et al., 2017) and produce more ROS species in the aggregate state (Gu et al., 2017). These properties of AIE-based PSs make them a better choice for imageguided PDT and tumor-killing (Wu et al., 2017a). However, PSs with NIR emissions and high ROS production still remain to be explored. Wu et al. (2017b) reported the fabrication of a kind of PS TPETCAQ (Figure 5A) with AIE characteristics for NIR image-guided PDT. Encapsulation of TPETCAQ using a DSPE-PEG-MAL matrix and subsequent surface modification with the HIV-1 transactivator were used to obtain the final product, TPETCAQ NPs (Figure 5B). The resultant product exhibited strong fluorescence emission intensity at $820 \mathrm{~nm}$, accompanied by much more ${ }^{1} \mathrm{O}_{2}$ production than $\mathrm{Ce} 6$, a wellknown highly efficient PSs. The bioluminescence of tumor in vivo progressively reduced with time, which demonstrated that the nano-PSs were efficient for image-guided PDT. Also, only very faint luminescence signals were detected from the AIE-based PSs after $14 \mathrm{~d}$, suggesting their high PDT efficacy (Figure 5C). The excellent PDT efficacy of the AIE PSs was further confirmed by tumor volume changes for different groups (Figure 5D).

In addition to low toxicity, photobleaching resistance, and deep tissue penetration, UCNPs also have the unique properties of transforming from NIR emission to visible light ( $\mathrm{Gu}$ et al., 2013; Chan et al., 2015). Notably, the long-lived red emissions from UCNPs overlap partly with the absorptions of some PSs, e.g., ZnPc or Ce6, which offers a valid energy transfer pathway to activate PSs for PDT under NIR irradiation (Idris et al., 2015; Tian et al., 2015). Huang et al. (2016) prepared $\mathrm{Na}_{0.52} \mathrm{YbF}_{3.52}$ :Er UCNPs with intense red emission using a simple solvothermal approach for multimodal imaging and tumor PDT. A low $[\mathrm{Na}] /[\mathrm{Yb}]$ ratio in UNCPs was found, owing to the lack of elemental $\mathrm{Na}$, which resulted in high luminescence intensity and color purity for red UC emission of $\mathrm{Er}^{3+}$. The UNCPs displayed dominant red and weak green UC emission at 655 and $522 / 543 \mathrm{~nm}$, which were attributed to the intra-4f transitions of $\mathrm{Er}^{3+},{ }^{4} \mathrm{~F}_{9 / 2} \rightarrow{ }^{4} I_{15 / 2}$, and ${ }^{2} \mathrm{H}_{11 / 2} /{ }^{4} S_{3 / 2} \rightarrow{ }^{4} I_{15 / 2}$, respectively. In addition, a thin $\mathrm{SrF}_{2}$ layer was modified on the UNCPs, and the red UC emission intensity was increased by about 17 times, while a relatively high intensity ratio $(\sim 5.8)$ of Red/Green was still retained. To make them utilizable in vivo, the $\mathrm{Na}_{0.52} \mathrm{YbF}_{3.52}$ :Er@SrF $\mathrm{SrNP}_{2}$ UCNere further modified with DSPE-PEG to obtain the final low toxicity product Lipo-UCNPs. Significantly, the PS ZnPc-loaded Lipo-UCNPs exhibited high efficacy in ${ }^{1} \mathrm{O}_{2}$ production and cancer cell killing under $915 \mathrm{~nm}$ irradiation, avoiding the overheating effect usually resulting from $980 \mathrm{~nm}$ excitation. After intratumoral injection of $50 \mu \mathrm{L} \mathrm{ZnPc-}$ Lipo-UCNPs $\left(10 \mathrm{mg} \mathrm{mL}^{-1}\right)$ into HeLa tumor-bearing mice, the tumor volumes increased much more slowly, demonstrating the significant tumor growth rate inhibition effect of the 
TABLE 2 | Examples of PA contrast agent explored in PA imaging.

\begin{tabular}{|c|c|c|c|c|}
\hline Materials & & Types of nanoagents & Advantages (+)/Disadvantages (-) & References \\
\hline \multirow[t]{3}{*}{ Inorganic } & Metallic nanomaterials & $\begin{array}{l}\text { Au nanorods; } \\
\text { Au nanostars; } \\
\text { Au nanocages; } \\
\text { Au nanoshell; } \\
\text { Au nanovesicles; } \\
\text { Au nanoflowers; } \\
\text { Ag nanoplates; } \\
\text { Palladium nanoplates; } \\
\text { antimony nanoparticles }\end{array}$ & $\begin{array}{l}\text { (+) tunable physiochemical } \\
\text { properties; } \\
\text { chemically inert element with } \\
\text { reasonable } \\
\text { biocompatibility; able to carry } \\
\text { cargoes. } \\
\text { (-) non-biodegradability; } \\
\text { suboptimal photothermal stability }\end{array}$ & $\begin{array}{l}\text { Chen et al., 2015b; Zhong et al., } \\
2015 \\
\text { Wang et al., 2015a } \\
\text { Zhang et al., } 2013 \\
\text { Topete et al., } 2014 \\
\text { Song et al., 2015a } \\
\text { Huang et al., 2014a } \\
\text { Homan et al., } 2012 \\
\text { Nie et al., } 2014 \\
\text { Li and Chen, } 2015\end{array}$ \\
\hline & Carbon-based nanomaterials & $\begin{array}{l}\text { Carbon nanotubes; } \\
\text { Graphenes; } \\
\text { Carbon dots }\end{array}$ & $\begin{array}{l}(+) \text { able to carry cargoes; } \\
\text { good photothermal stability. } \\
(-) \\
\text { non-biodegradability; heterogeneity }\end{array}$ & $\begin{array}{l}\text { Zhang et al., } 2015 \\
\text { Lalwani et al., 2013; Sheng et al., } \\
\text { 2013; Ge et al., } 2015\end{array}$ \\
\hline & $\begin{array}{l}\text { Transition metal chalcogenides } \\
\text { (TMC)-based nanomaterials }\end{array}$ & $\begin{array}{l}\text { CuS; } \mathrm{WS}_{2} ; \mathrm{MoS}_{2} ; \\
\text { FeS; } \mathrm{Bi}_{2} \mathrm{~S}_{3} ; \text { CuSe; } \\
\mathrm{Co}_{9} \mathrm{Se}_{8} ; \mathrm{Bi}_{2} \mathrm{Se}_{3}\end{array}$ & $\begin{array}{l}\text { (+) high photothermal conversion } \\
\text { efficiency; } \\
\text { good photothermal stability; low cost. } \\
\text { (-) non-biodegradability; } \\
\text { contain heavy metal elements }\end{array}$ & $\begin{array}{l}\text { Cheng et al., 2014a; Yin et al., } 2014 \\
\text { Cui et al., 2015; Yang et al., 2015b } \\
\text { Hessel et al., 2011; Liu et al., 2015e } \\
\text { Song et al., 2015b }\end{array}$ \\
\hline \multirow[t]{2}{*}{ Organic } & Dyes & $\begin{array}{l}\text { Porphyrin- and } \\
\text { Cyanine-based dyes, } \\
\text { e.g., Indocyanine green (ICG), IR780, } \\
\text { IR825, etc. }\end{array}$ & $\begin{array}{l}\text { (+) good } \\
\text { biocompatibility/biodegradability. } \\
(-) \text { poor aqueous solubility, } \\
\text { low photothermal stability, } \\
\text { short bloodstream circulation half-life }\end{array}$ & $\begin{array}{l}\text { Sheng et al., 2014; } \\
\text { Wang et al., 2015b } \\
\text { Lovell et al., 2011; Song et al., 2015c } \\
\text { Huang et al., 2014b; Chen et al., } \\
\text { 2015a; Rong et al., } 2015\end{array}$ \\
\hline & Polymer-based nanomaterials & $\begin{array}{l}\text { Polypyrrole; } \\
\text { Polyaniline; } \\
\text { Polydopamine; } \\
\text { Semiconducting } \\
\text { polymers }\end{array}$ & $\begin{array}{l}(+) \text { good biocompatibility and } \\
\text { photothermal stability; able to carry } \\
\text { cargoes. } \\
(-) \text { their biodegradation behaviors } \\
\text { remain unknown }\end{array}$ & $\begin{array}{l}\text { Yang et al., 2011; Lin et al., } 2014 \\
\text { Yang et al., 2012; Pu et al., } 2015\end{array}$ \\
\hline
\end{tabular}

UNCP-based PDT. The results revealed the great potential of UCNPs with high-purity red emission for imaging-guided PDT of tumors.

Most PSs for PDT possess poor solubility in water and suboptimal selectivity in vivo (Yan et al., 2015). To overcome these limitations, PSs are usually conjugated with nanocarriers with a high surface area, e.g., GO (Yang et al., 2013). Various PSs have been designed to load on the surface of GO via $\pi$ $\pi$ stacking and hydrophobic forces for tumor imaging and PDT in animal models (Rong et al., 2014; Shi et al., 2014). However, most PS-GO nanoconjugates passively targeted to tumors in vivo via an EPR effect. Thereby, some tumor-specific molecules (e.g., peptides, ligands, and antibodies) need to be modified onto GO to increase targeting and PDT efficacy (Shi et al., 2015b). Yu et al. (2017) fabricated a PS-loaded GO nanocomplex by conjugating PEGylated GO with a tumorselective HK peptide that can specifically bind to highly expressed integrin $\alpha v \beta 6$ receptor in many tumor types and following functionalization with a PS. The tumor uptake of GO(PS)-PEGHK was obviously higher than that of free PS and GO(PS)-PEG, demonstrating the specific intake of the nanocomplex in tumors by the recognition of integrin $\alpha v \beta 6$. Tumor recurrence, especially lung metastasis, was significantly inhibited in mice for nearly a month by vaccination with necrotic $4 \mathrm{~T} 1$ tumor cells induced by the nanocomplex PDT. The nanocomplex-treated necrotic tumor cells could activate dendritic cells and significantly inhibit tumor growth and lung metastasis by increasing the infiltration of cytotoxic $\mathrm{CD}^{+}{ }^{+} \mathrm{T}$ lymphocytes in tumor. Using the nanocomplex, the primary tumor, and the residual tumor cells could be efficiently killed by activating host anti-tumor immunity and stimulating the immune memory, accordingly prohibiting distant metastasis. These findings suggest that nanocomplexbased PDT is an effective strategy for eliminating residual cells after tumor resection and preventing tumor recurrence and distant metastasis.

\section{Photothermal Therapy (PTT)}

Photothermal therapy (PTT) utilizes the photothermal effect of a photothermal transduction agent (PTA) that can harvest the energy from light and transform that energy into heat to raise the surrounding temperature and induce the death of cancer cells. An ideal PTA should possess high photothermal conversion efficiency (PCE), strong absorption in the NIR region, and good accumulation in tumors without toxic side effects. In particular, nano PTAs can accumulate in tumors through the EPR effect and active targeting. Also, nano PTAs can realize higher PCE than small-molecule PTAs and can potentially be used with multiple imaging modalities and incorporate various therapeutic functions for advanced application (Cheng et al., 2014b). Nano PTAs can be divided into inorganic and organic materials (Gai et al., 2018). Inorganic materials include noble metals (Riley and Day, 2017), metal chalcogenides (Li et al., 2017b), carbon-based 


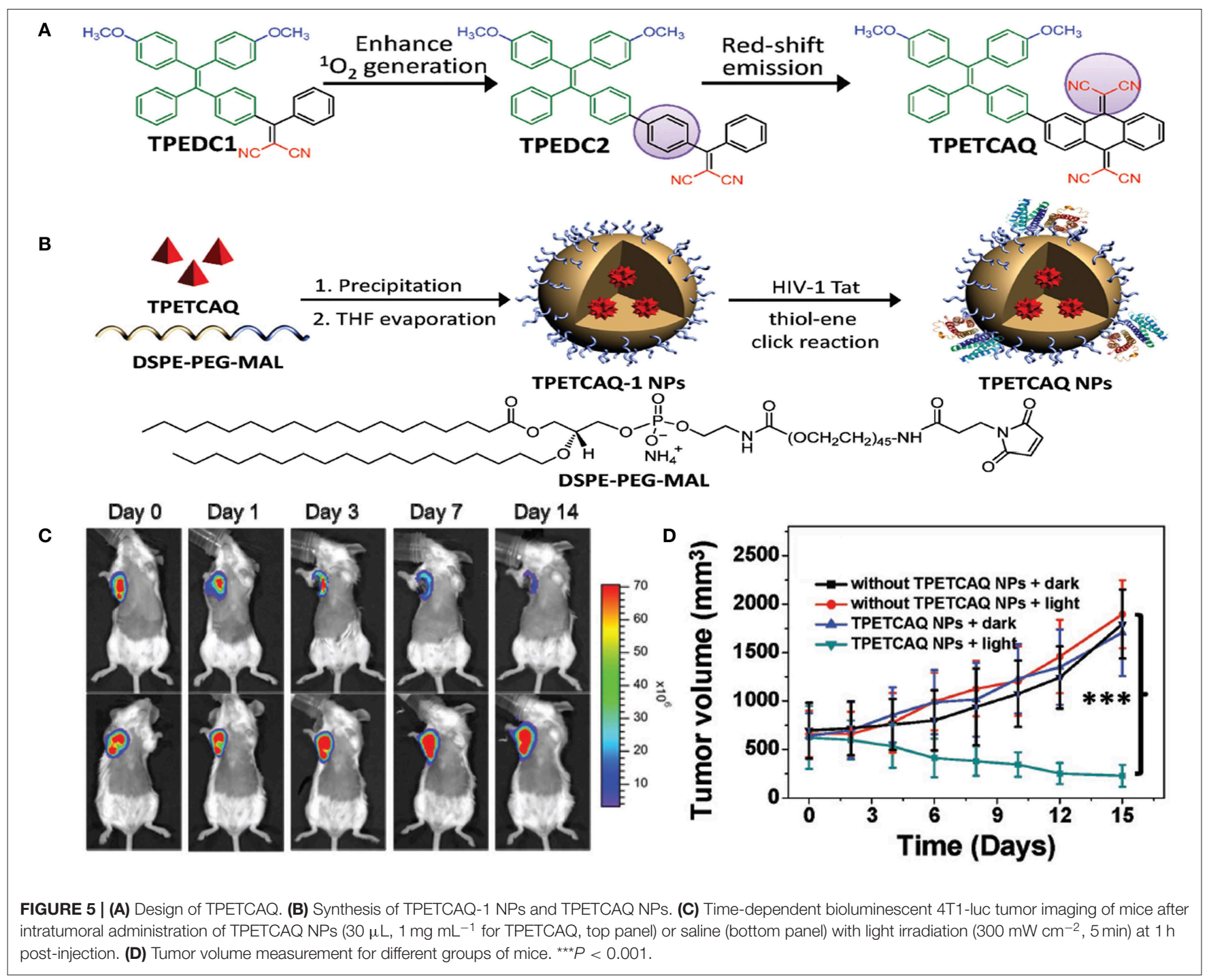

materials (Hong et al., 2015), and other two-dimensional (2D) materials (e.g., black phosphorus, nanosheets, boron nitride, graphitic carbon nitride, MXenes) (Chen et al., 2015c; Augustine et al., 2017; Tan et al., 2017; Choi et al., 2018; Huang et al., 2018). Organic PTAs include semiconducting polymer NPs (SPNPs), nanomicelle-encapsulated NIR dyes, and porphysomes (Jung et al., 2018; Liu et al., 2019b).

Recently, stoichiometric semiconductor metal sulfide nanocrystals (e.g., $\mathrm{Ag}_{2} \mathrm{~S}$ and $\mathrm{CuS}$ ) have been developed for optical imaging and PTT due to their strong absorption capacity in the NIR-region, negligible photobleaching, high photoconversion efficiency, ultrasmall size, and good inertia (e.g., the solubility product constant is $K_{\mathrm{sp}}=6.3 \times 10^{-50}$ for $\mathrm{Ag}_{2} \mathrm{~S}$ ) (Hong et al., 2012b). Yang et al. (2017b) designed dimension-dependent $\mathrm{Ag}_{2} \mathrm{~S}$ nanodots (NDs) as a photothermal agent for PTT and multimodal imaging. These NDs are prepared by finely modulating their growth within clinically acceptable HSA nanocages (Figure 6A). They exhibited a narrow emission band (full-width at half-maximum $\sim 8.0 \mathrm{~nm}$ ) due to their monodisperse size distribution (Figure 6B). Also, larger NDs showed a higher fluorescence response in the NIR-II range, suggesting that the fluorescence spectrum strongly depended upon their diameters. $\mathrm{Ag}_{2} \mathrm{~S}-\mathrm{NDs}$ also displayed obvious size-dependent temperature elevations (Figure 6C), mainly originating from their size-dependent molar extinction coefficients. Additionally, strong non-radiative energy decay in the conduction band may contribute to the efficient photothermal conversion efficacy of the NDs. The improved circulation and EPR effect of NDs caused them to be mainly distributed into tumor, suggesting their high uptake in tumor (Figure 6D). The NDs showed remarkable fluorescence within tumor at $48 \mathrm{~h}$ after injection, whereas the control group displayed a faint signal due to fast elimination of NDs (Figure 6E). These findings indicated that the NDs could be accumulated at the tumor site for a long time, which was beneficial to flexible imaging or PTT. The NDs showed time-dependent uptakes in other tissues, including heart, liver, spleen, lung, and kidney. Moreover, the NDs were progressively excluded from the tissues 


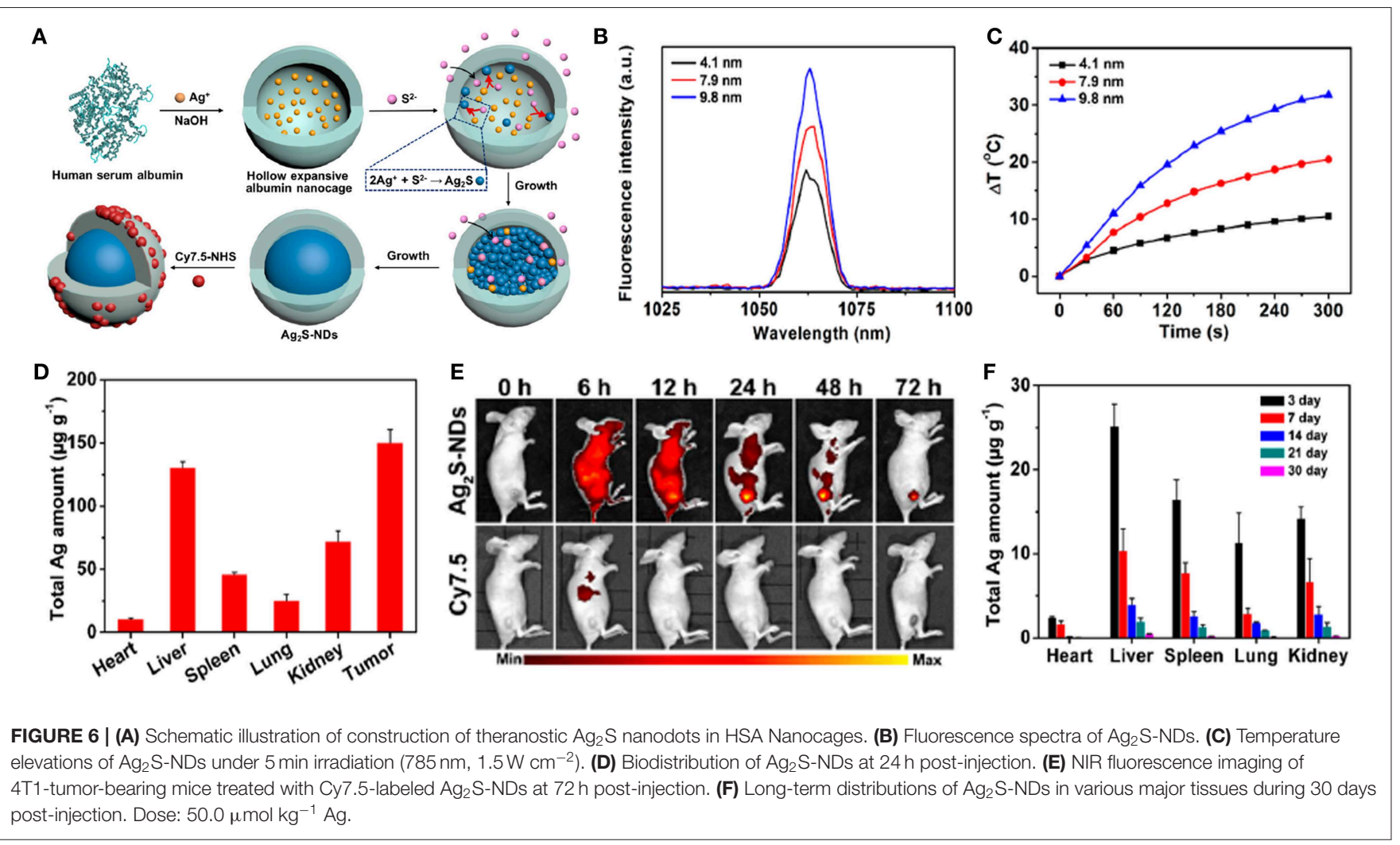

in 30 days via renal excretion (Figure 6F), thus avoiding potential toxicity concerns.

Copper chalcogenide $\left(\mathrm{Cu}_{2-n} \mathrm{R}, \mathrm{R}=\mathrm{S}\right.$, Se, Te, $0 \leq \mathrm{n} \leq$ 1) NPs, which possess strong localized SPRs (LSPRs) in the NIR region due to copper deficiency, have been employed for PA imaging and PTT (Coughlan et al., 2017). $\mathrm{Cu}_{2-n} \mathrm{R}$ NPs may be much smaller than conventional photothermal agents such as GNPs (typically $>50 \mathrm{~nm}$ in 1D to generate NIR LSPR). Also, their good biodegradability allows the release of the vital trace elements copper and chalcogen to maintain the health of the organism (for example, selenium or copper deficiency contributes to the incidence and mortality of some cancers [e.g., liver, prostate, and lung)] or the evolution and aggravation of some cardiovascular diseases and diabetes (Zhou et al., 2016b). Monodisperse $\mathrm{Cu}_{2-n} \mathrm{Se}$ NPs are usually synthesized in organic solvents at high temperature, and they then need laboriously subsequent modification to make them utilizable in biological fields (van der Stam et al., 2015; Yan et al., 2017). Zhang et al. (2016) prepared novel ultrasmall PEGylated $\mathrm{Cu}_{2-n} \mathrm{Se}$ $\mathrm{NP}$ in aqueous solution for multimodal imaging-guided tumor PTT. The $\mathrm{Cu}_{2-n} \mathrm{Se}$ NPs were synthesized in distilled water and formed by a characteristic dark green solution and were then functionalized with dimercaptosylated PEG to enhance their solubility, dispersity, and biocompatibility after ultrafiltration. The final product displayed strong LSPRs in the NIR region $(600-1,100 \mathrm{~nm})$ owing to the high hole density due to copper deficiency. The high extinction coefficient $\left(8.5 \mathrm{Lg}^{-1} \mathrm{~cm}^{-1}\right)$ of the PEGylated $\mathrm{Cu}_{2-n}$ Se NPs at $808 \mathrm{~nm}$ reflected a distinct photothermal conversion capability. Also, the NPs showed good photothermal stability and long-term circulation with a halflife of $8.14 \mathrm{~h}$ owing to the presence of water-soluble and large sterically hindered PEG. After intravenous injection of the PEGylated $\mathrm{Cu}_{2-n}$ Se NPs into BALB/c mice bearing 4T1 tumors (130 $\mathrm{mm}^{3}$ volume), the tumor temperature of the mice rose steeply to $57.6^{\circ} \mathrm{C}$ during NIR irradiation, which was high enough to kill the cancer cells and to halt their lethal proliferation. The tumors shrank and became black scars at day 3 and were removed totally in $16 \mathrm{~d}$ without recurrence. These NPs might become an effective PTT agent for in vivo tumor therapy.

Nanodiamonds (NDDs) with diameters of $2-10 \mathrm{~nm}$ and a truncated octahedral framework have appeared as innovative materials for bioimaging and therapy due to their good biocompatibility, spherical morphology, high density, large surface area, and surface functionality (Mochalin et al., 2011). Ryu et al. (2016) designed folic acid (FA)-conjugated NDD (FA-NDD) nanoclusters by using the unique features of NDDs for PTT. FA was selected as a model targeting ligand for tumor and receptor-mediated endocytosis since its receptors are generally overexpressed in some types of tumor cells. Their spherical morphology and good biocompatibility enabled NDDs to act as a nanoplatform for delivery systems. Amination of NDD nanoclusters with - $\mathrm{COOH}$ via 1,2-ethylenediamine and subsequent modification with FA by carbodiimide chemistry were performed. After 5-min laser irradiation, the temperature of NDD $\left(10 \mu \mathrm{g} \mathrm{mL}^{-1}\right)$ rose to $54^{\circ} \mathrm{C}$. Compared with WI-38 cells (negative control), FA-NDD nanoclusters easily entered the KB 

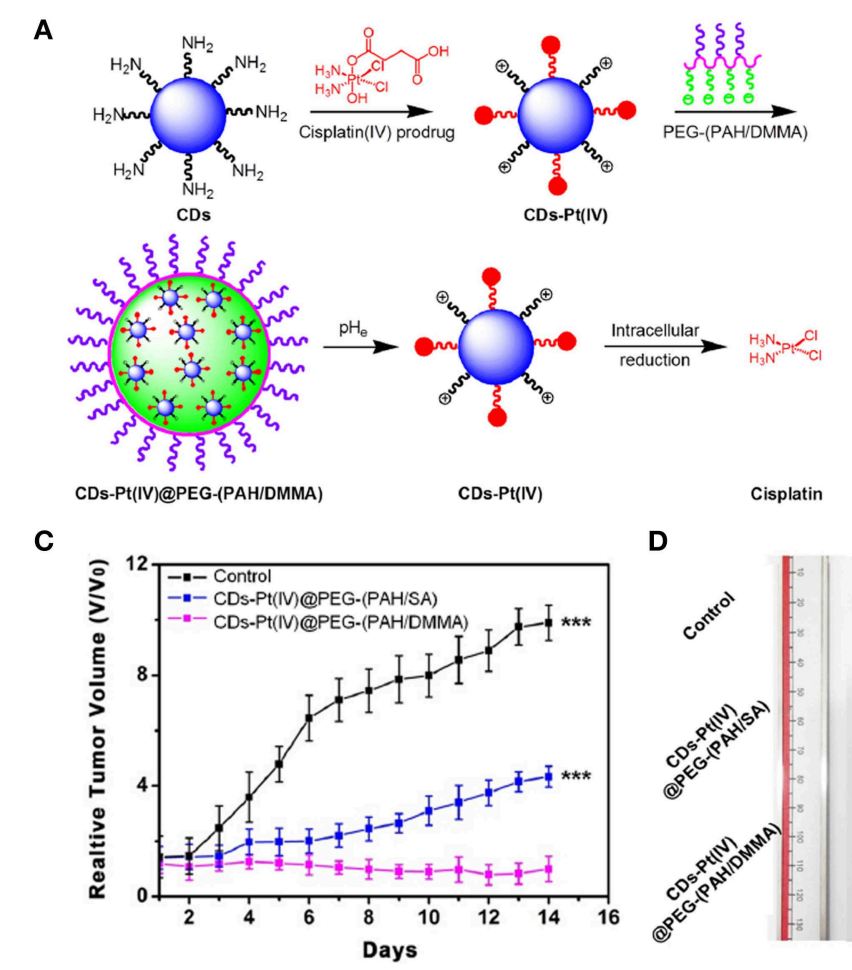

D

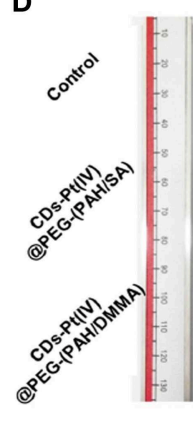

B

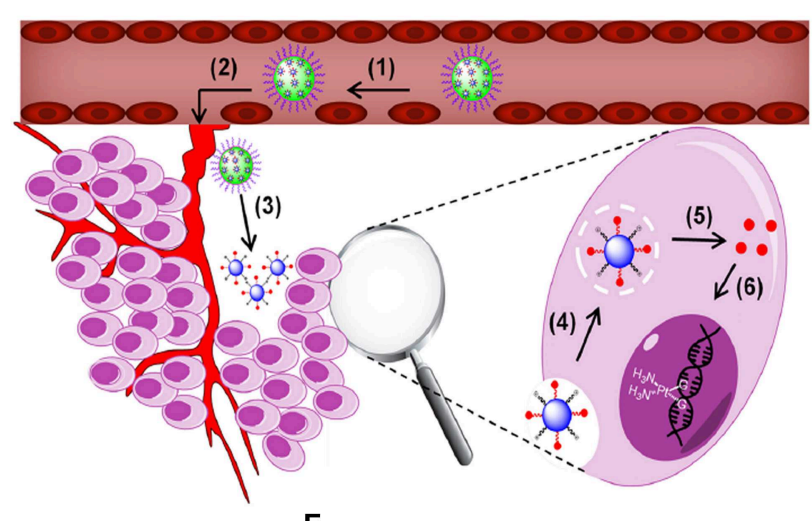

E

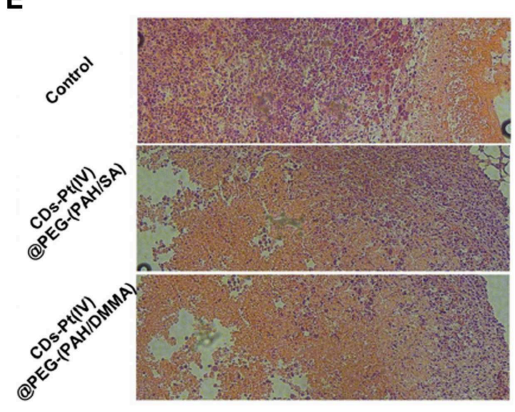

FIGURE 7 | (A) Schematic illustration for the preparation of charge-convertible CD-based drug nanocarrier. pHe means tumor extracellular pH. (B) Schematic illustration for the drug delivery process: (1) negative charge/PEGylation to prolong circulation time, (2) accumulation at the tumor site through the EPR effect, (3) responsiveness to tumor extracellular $\mathrm{pH}$, (4) effective uptake by cancer cells, (5) facilitated endosome escape by the "proton sponge" effect and controlled cisplatin release, and (6) cisplatin binding with DNA to exhibit cytotoxicity. (C) Relative tumor volume achieved from mice after intravenous treatments with CD-based drug nanocarrier. (D) Photographs of mice and excised tumors from representative euthanized mice. (E) H\&E stained tumor slices from different groups after 14-day treatment. ${ }^{\star \star *} P<0.001$.

cells (positive control), indicating specificity to tumor cells that overexpress FA receptors. Cell viability assay and fluorescence microscopic imaging clearly showed that FA-NDD preferred to ablate $\mathrm{KB}$ cells selectively rather than WI-38 cells under NIR laser illumination. For tumor-bearing nude mice, a substantial accumulation of FA-NDD in tumor led to a significant decrease in tumor volume, and almost absent $14 \mathrm{~d}$ after NIR laser exposure as compared to NDD nanoclusters. These results clearly verified that the combination of the FA-NDD nanoclusters and NIR light might be an efficient and feasible tumor therapy.

\section{Optical-Responsive Drug Delivery}

The effective delivery and release of drugs to targets remains a great challenge to improving therapies for human diseases (Devadasu et al., 2013). A recognized strategy is to construct a target-specific drug delivery system (DDS) that can carry an efficient dosage of the drug to targeted cells and tissues (Wong and Choi, 2015). An ideal stimuli-responsive DDS should possess the following features: biocompatible or biodegradable composition, high drug-loading capacity, a site-specific delivery mechanism to spare normal cells and tissues, no premature drug release, and accurate release in response to exogenous or endogenous stimulus (Karimi et al., 2016; Liang et al., 2016). Various nanocarriers such as liposomes (Grimaldi et al., 2016), polymers (Nicolas et al., 2013; Tong et al., 2014), micelles (Cabral et al., 2018), dendrimers (Astruc et al., 2010), silica (Wen et al., 2017), gold NPs (Arvizo et al., 2012), black phosphorus (Qiu et al., 2019), and carbon-based nanomaterials (Panwar et al., 2019) have been developed for medical purposes. Robust and effective nanocarriers could even be responsive to multiplex combinations of diverse stimuli to further strengthen their specificity for targeted and controlled drug delivery (Biju, 2014).

CDs can serve as imaging probes or as nanocarriers for transporting the targeted theranostic agents (e.g., PSs, drugs, genes) (Boakye-Yiadom et al., 2019). The surfaces of CDs, as the drug nanocarriers, are usually modified by negatively or positively charged PEG, which affects their therapeutic efficacy and future biological application. Owing to electrostatic repulsion from the same-charged cell membrane, negatively charged PEGylated CDs nanocarriers cannot easily enter the cancer cells, which affects intake and ultimately leads to low curative effects. Due to non-specific interactions with cellular ingredients (e.g., serum), positively charged CD carriers can be easily endocytosed through RES, resulting in quick clearance from blood circulation. Also, positively charged nanocarriers can be phagocytized by healthy cells to produce some potential toxicity via charge attraction with cell 
membrane (Mishra et al., 2018). To solve the above-mentioned problems, Feng et al. (2016) developed a cisplatin-loaded charge convertible $\mathrm{CD}$ nanocarrier functionalized by poly(allyamine)-modified PEG, which could sensitively distinguish the $\mathrm{pH}$ difference between tumor pathologic outcomes and the normal physiological environment for controllable drug delivery (Figure 7A). The anionic poly-(allyamine)-modified PEG polymer with dimethylmaleic acid on the constructed CD nanocarrier would experience a charge transformation into the cationic polymer under the mild acidity of tumor cells $(\mathrm{pH} \sim 6.8$ ), resulting in intense electrostatic repulsion and "leakage" of cisplatin(IV)-CD cations. Significantly, positive cisplatin(IV)-CDs showed high affinity for negative cancer cell membrane, thus leading to the enhancement of internalization and efficient release of drug in the reductive cytosol (Figure 7B). Tumors growth in mice injected with the drug-loaded CDs were greatly suppressed during 14 days of treatment (Figure 7C). The digital images of tumor excision in mice further confirmed that the sizes of the tumors were smallest using the drug-loaded CDs, indicating their better treatment efficiency (Figure 7D). Also, through hematoxylin and eosin $(\mathrm{H} \& \mathrm{E})$ staining analysis of tumor tissues (Figure 7E), the drug-loaded $\mathrm{CD}$ treatment groups exhibited the greatest degree of cell injury, which was consistent with the tumor growth data.

Zinc gallogermanate structured PLNPs have demonstrated superior physiochemical properties, e.g., a strong NIR signal (quantum yield $\sim 10 \%$ ), superlong optical lifetime ( $>90 \mathrm{~h}$ ), monodisperse stability, and minimal poisoning (Abdukayum et al., 2013). The superlong sustained emission and red light renewability of the trivalence-doped zinc gallogermanate PLNPs (ZGGO: $\mathrm{Cr}^{3+}, \mathrm{Yb}^{3+}, \mathrm{Er}^{3+}$ ) are the principal basis for long circulation bioimaging and drug release in living organisms (Li and Yan, 2016). Also, the easily controllable surface modulation of ZGGO PLNPs makes them desirable alternatives for fabricating the drug carriers. Liu et al. (2018b) incorporated red blood cell membrane vesicles with NIR PLNPs to construct an erythrocyte membrane bioexcited optical nanocarrier. In order to realize the biomimetic pattern, erythrocyte membrane vesicles were isolated from $\mathrm{RBC}$ and then fused with mesoporous $\mathrm{SiO}_{2}$ coated ZGGO to form the membrane bioexcited nanocarriers. Three groups of 4T1 tumor-bearing mice were individually treated with unmodified, $\mathrm{SiO}_{2}$-coated, or membrane-fused ZGGO via tail vein injection. The NPs were mainly distributed in the two primary RES organs (i.e., liver and spleen) and the tumors due to EPR effects. Meanwhile, compared with two other NPs, the membrane-fused ZGGO exhibited a far higher proportion of retention capacity. Moreover, the amounts of membranefused ZGGO loaded with the drug Dox in blood were measured,

\section{REFERENCES}

Abdukayum, A., Chen, J. T., Zhao, Q., and Yan, X. P. (2013). Functional near infrared-emitting $\mathrm{Cr}^{3+} / \mathrm{Pr}^{3+}$ co-doped zinc gallogermanate persistent luminescent nanoparticles with superlong afterglow for in vivo targeted bioimaging. J. Am. Chem. Soc. 135, 14125-14133. doi: 10.1021/ja404243v and the results indicated a long cycling period with a half-life of $9 \mathrm{~h}$. Furthermore, injection of the drug-delivery vehicle into mice exhibited improved sustained-release efficacy of the NPs, strongly confirming the efficient decrease of systemic clearance and extension of circulation time caused by the biomimetic membrane coating. Employment of the drug-delivery system in the $4 \mathrm{~T} 1$ orthotopic mammary tumor model showed the best tumor growth inhibition performance.

\section{SUMMARY AND PERSPECTIVES}

By conjugating with different functional groups, ligands, and biomolecules, multifunctional NPs have displayed superb capabilities in diagnosis and therapeutic applications. Diverse designs and synthesis strategies have been developed to achieve specific NPs offering targeted and controlled drug delivery for practical use. Multiple aspects of NP research, from investigations of the effects of different kinds of NPs and their sizes on the imaging and therapeutic efficacy in biological environments to studies of specific ligand targeted drug delivery, have been carefully performed.

Despite these exciting achievements in the last few years, the distinctive functions of multifunctional nanomaterials have influenced the injection dose in different imaging models and therapy types. Therefore, the issues around the controllable synthesis of nanoagents, synergistic theranostic effects, and short-term and long-term toxicity should be carefully investigated as a whole to realize every function (diagnosis and therapy) and to achieve the lowest side effect with single injection.

More investigations are required to improve the performance of theranostic agents, such as intensifying excretion, strengthening continuous monitoring, prolonging blood circulation time, promoting physiological barrier penetration, evading the RES, and accelerating more materials into clinical trials. Therefore, continuous innovations and development for novel theranostic approaches are necessary to meet growing clinical requirements.

\section{AUTHOR CONTRIBUTIONS}

YY designed the whole structure of the article, wrote the sections on materials, bio-imaging, PDT, and reviewed the article. LW, BW, YG, and XL wrote the sections on PTT and drug delivery.

\section{FUNDING}

This work was supported by the National Natural Science Foundation of China (Nos. 21577163, 21377142, 21477146). 
Arvizo, R. R., Bhattacharyya, S., Kudgus, R. A., Girl, K., Bhattacharya, R., and Mukherjee, P. (2012). Intrinsic therapeutic applications of noble metal nanoparticles: past, present and future. Chem. Soc. Rev. 41, 2943-2970. doi: $10.1039 / \mathrm{c} 2 \mathrm{cs} 15355 \mathrm{f}$

Astruc, D., Boisselier, E., and Ornelas, C. (2010). Dendrimers designed for functions: from physical, photophysical, and supramolecular properties to applications in sensing, catalysis, molecular electronics, photonics, and nanomedicine. Chem. Rev. 110, 1857-1959. doi: 10.1021/cr9 $00327 \mathrm{~d}$

Augustine, S., Singh, J., Srivastava, M., Sharma, M., Das, A., and Malhotra, B. D. (2017). Recent advances in carbon based nanosystems for cancer theranostics. Biomater. Sci. 5, 901-952. doi: 10.1039/C7BM00008A

Auzel, F. (2004). Upconversion and anti-stokes processes with $\mathrm{f}$ and $\mathrm{d}$ ions in solids. Chem. Rev. 104, 139-173. doi: 10.1021/cr020357g

Bao, S. J., Huang, S. N., Liu, Y., Hu, Y. R., Wang, W. P., Ji, M. F., et al. (2017). Gold nanocages with dual modality for image-guided therapeutics. Nanoscale 9, 7284-7296. doi: 10.1039/C7NR01350G

Bartelmess, J., Quinn, S. J., and Giordani, S. (2015). Carbon nanomaterials: multifunctional agents for biomedical fluorescence and Raman imaging. Chem. Soc. Rev. 44, 4672-4698. doi: 10.1039/C4CS00306C

Baumberg, J., Bell, S., and Bonifacio, A. (2017). SERS in biology/biomedical SERS: general discussion. Faraday Discuss. 205, 429-456. doi: 10.1039/C7FD90089A

Bechet, D., Couleaud, P., Frochot, C., Viriot, M. L., Guillemin, F., and Barberi-Heyob, M. (2008). Nanoparticles as vehicles for delivery of photodynamic therapy agents. Trends Biotechnol. 26, 612-621. doi: 10.1016/j.tibtech.2008.07.007

Bessiere, A., Jacquart, S., Priolkar, K. R., and Lecointre, A. (2011). $\mathrm{ZnGa}_{2} \mathrm{O}_{4}: \mathrm{Cr}^{3+}$ : a new red long-lasting phosphor with high brightness. Opt. Exp. 19, 10131-10137. doi: 10.1364/OE.19.010131

Biju, V. (2014). Chemical modifications and bioconjugate reactions of nanomaterials for sensing, imaging, drug delivery and therapy. Chem. Soc. Rev. 43, 744-764. doi: 10.1039/C3CS60273G

Bilan, R., Nabiev, I., and Sukhanova, A. (2016). Quantum dot-based nanotools for bioimaging, diagnostics, and drug delivery. Chembiochem 17, 2103-2114. doi: $10.1002 /$ cbic. 201600357

Bimbo, L. M., Sarparanta, M., Santos, H. A., Airaksinen, A. J., Makila, E., Laaksonen, T., et al. (2010). Biocompatibility of thermally hydrocarbonized porous silicon nanoparticles and their biodistribution in rats. ACS Nano 4, 3023-3032. doi: 10.1021/nn901657w

Birel, O. (2017). A review on perylene-3,4,9,10-tetracarboxylic acid diimide molecules. CBU J. Sci. 13, 379-386. doi: 10.18466/cbayarfbe.319879

Boakye-Yiadom, K. O., Kesse, S., Opoku-Damoah, Y., Filli, M. S., Aquib, M., Joelle, M. M. B., et al. (2019). Carbon dots: applications in bioimaging and theranostics. Int. J. Pharmaceut. 564, 308-317. doi: 10.1016/j.ijpharm.2019.04.055

Boisselier, E., Diallo, A. K., Salmon,. L., Ornelas, C., Ruiz, J., and Astruc, D. (2010). Encapsulation and stabilization of gold nanoparticles with"click" polyethyleneglycol dendrimers. J. Am. Chem. Soc. 132, 2729-2742. doi: 10.1021/ja909133f

Boisselier, E., Salmon, L., Ruiz, J., and Astruc, D. (2008). How to very efficiently functionalize gold nanoparticles by "click" chemistry. Chem. Commun. 30, 5788-5790. doi: 10.1039/b812249k

Brigger, I., Dubernet, C., and Couvreur, P. (2002). Nanoparticles in cancer therapy and diagnosis. Adv. Drug Delivery Rev. 54, 631-651. doi: 10.1016/S0169-409X(02)00044-3

Brust, M., Walker, M., Bethell, D., Schiffrin, D. J., and Whyman, R. (1994). Synthesis of thiol-derivatised gold nanoparticles in a two-phase liquid-liquid system. J. Chem. Soc. 7, 801-802. doi: 10.1039/C39940 000801

Bullock, C. J., and Bussy, C. (2019). Biocompatibility considerations in the design of graphene biomedical materials. Adv. Mater. Interfaces 6:1900229. doi: 10.1002/admi.201900229

Cabral, H., Miyata, K., Osada, K., and Kataoka, K. (2018). Block copolymer micelles in nanomedicine applications. Chem. Rev. 118, 6844-6892. doi: 10.1021/acs.chemrev.8b00199

Cai, Y., Wei, Z., Song, C. H., Tang, C. C., Han, W., and Dong, X. C. (2019). Optical nano-agents in the second near-infrared window for biomedical applications. Chem. Soc. Rev. 48, 22-37. doi: 10.1039/C8CS00494C
Castillo, R. R., and Vallet-Regi, M. (2019). Functional mesoporous silica nanocomposites: biomedical applications and biosafety. Int. J. Mol. Sci. 20:929. doi: 10.3390/ijms20040929

Cha, M. G., Lee, S., Park, S., Kang, H., Lee, S. G., Jeong, C., et al. (2017). A dual modal silver bumpy nanoprobe for photoacoustic imaging and SERS multiplexed identification of in vivo lymph nodes. Nanoscale 9, 12556-12564. doi: 10.1039/C7NR03742B

Chan, E. M., Levy, E. S., and Cohen, B. E. (2015). Rationally designed energy transfer in upconverting nanoparticles. Adv. Mater. 27, 5753-5761. doi: 10.1002/adma.201500248

Chang, C. C., Hsieh, M. C., Lin, J. C., and Chang, T. C. (2012). Selective photodynamic therapy based on aggregation-induced emission enhancement of fluorescent organic nanoparticles. Biomaterials 33, 897-906. doi: 10.1016/j.biomaterials.2011.10.018

Chang, Y. R., Lee, H. Y., Chen, K., Chang, C. C., Tsai, D. S., Fu, C. C., et al. (2008). Mass production and dynamic imaging of fluorescent nanodiamonds. Nat. Nanotechnol. 3, 284-288. doi: 10.1038/nnano.2008.99

Chen, D. Q., Chen, Y., Lu, H. W., and Ji, Z. G. (2014). A bifunctional $\mathrm{Cr} / \mathrm{Yb} / \mathrm{Tm}: \mathrm{Ca}_{3} \mathrm{Ga}_{2} \mathrm{Ge}_{3} \mathrm{O}_{12}$ phosphor with near-infrared long-lasting phosphorescence and upconversion luminescence. Inorg. Chem. 53, 8638-8645. doi: 10.1021/ic501238u

Chen, G. Y., Roy, I., Yang, C. H., and Prasad, P. N. (2016a). Nanochemistry and nanomedicine for nanoparticle-based diagnostics and therapy. Chem. Rev. 116, 2826-2885. doi: 10.1021/acs.chemrev.5b00148

Chen, J., Saeki, F., Wiley, B. J., Cang, H., Cobb, M. J., Li, Z. Y., et al. (2005a). Gold nanocages: bioconjugation and their potential use as optical imaging contrast agents. Nano Lett. 5, 473-477. doi: 10.1021/nl047950t

Chen, J., Wiley, B., Li, Z. Y., and Campbell, D. (2005b). Gold nanocages: engineering their structure for biomedical applications. Adv. Mater. 17, 2255-2261. doi: 10.1002/adma.200500833

Chen, L. J., Sun, S. K., Wang, Y., Yang, C. X., Wu, S. Q., and Yan, X. P. (2016b). Activatable multifunctional persistent luminescence nanoparticle/copper sulfide nanoprobe for in vivo luminescence imagingguided photothermal therapy. ACS Appl. Mater. Interfaces 8, 32667-32674. doi: 10.1021/acsami.6b10702

Chen, Q., Liang, C., Wang, C., and Liu, Z. (2015a). An imagable and photothermal "abraxane-like" nanodrug for combination cancer therapy to treat subcutaneous and metastatic breast tumors. Adv. Mater. 27, 903-910. doi: 10.1002/adma.201404308

Chen, W. H., Yang, C. X., Qiu, W. X., Luo, G. F., Jia, H. Z., Lei, Q., et al. (2015b). Multifunctional theranostic nanoplatform for cancer combined therapy based on gold nanorods. Adv. Healthc. Mater. 4, 2247-2259. doi: 10.1002/adhm.201500453

Chen, Y., Tan, C. L., Zhang, H., and Wang, L. Z. (2015c). Two-dimensional graphene analogues for biomedical applications. Chem. Soc. Rev. 44, 2681-2701. doi: 10.1039/C4CS00300D

Cheng, L., Liu, J. J., Gu, X., Gong, H., Shi, X. Z., Liu, T., et al. (2014a). PEGylated $\mathrm{WS}_{2}$ nanosheets as a multifunctional theranostic agent for in vivo dualmodal CT/photoacoustic imaging guided photothermal therapy. Adv. Mater. 26, 1886-1893. doi: 10.1002/adma.201304497

Cheng, L., Wang, C., Feng, L. Z., Yang, K., and Liu, Z. (2014b). Functional nanomaterials for phototherapies of cancer. Chem. Rev. 114, 10869-10939. doi: $10.1021 / \mathrm{cr} 400532 \mathrm{z}$

Choi, J. R., Yong, K. W., Choi, J. Y., Nilghaz, A., Lin, Y., Xu, J., et al. (2018). Black phosphorus and its biomedical applications. Theranostics 8, 1005-1026. doi: 10.7150/thno. 22573

Coughlan, C., Ibanez, M., Dobrozhan, O., Singh, A., Cabot, A., and Ryan, K. M. (2017). Compound copper chalcogenide nanocrystals. Chem. Rev. 117, 5865-6109. doi: 10.1021/acs.chemrev.6b00376

Cui, J. B., Jiang, R., Xu, S. Y., Hu, G. F., and Wang, L. Y. (2015). $\mathrm{Cu}_{7} \mathrm{~S}_{4}$ nanosuperlattices with greatly enhanced photothermal efficiency. Small 11, 4183-4190. doi: 10.1002/smll.201500845

Dai, W. B., Lei, Y. F., Zhou, J., Xu, M., Chu, L. L., Li, L., et al. (2017). Nearinfrared quantum-cutting and long-persistent phosphor $\mathrm{Ca}_{3} \mathrm{Ga}_{2} \mathrm{Ge}_{3} \mathrm{O}_{12}$ : $\operatorname{Pr}^{3+}$, $\mathrm{Yb}^{3+}$ for application in vivo bioimaging and dye-sensitized solar cells. J. Alloy. Compd. 726, 230-239. doi: 10.1016/j.jallcom.2017.08.002

de Chermont, Q. L., Chaneac, C., Seguin, J., Pelle, F., Maitrejean, S., Jolivet, J. P., et al. (2007). Nanoprobes with near-infrared persistent luminescence 
for in vivo imaging. Proc. Natl. Acad. Sci. U.S.A. 104, 9266-9271. doi: 10.1073/pnas.0702427104

de la Zerda, A., Prabhulkar, S., Perez, V. L., Ruggeri, M., Paranjape, A. S., Habte, F., et al. (2015). Optical coherence contrast imaging using gold nanorods in living mice eyes. Clin. Exp. Ophthalmol. 43, 358-366. doi: 10.1111/ceo.12299

Desai, N. (2016). "Nanoparticle albumin-bound paclitaxel (Abraxane ${ }^{\circledR}$ )," in Albumin in Medicine, eds M. Otagiri and V. Chuang (Singapore: Springer Press), 101-119. doi: 10.1007/978-981-10-2116-9_6

Devadasu, V. R., Bhardwaj, V., and Kumar, M. N. V. R. (2013). Can controversial nanotechnology promise drug delivery? Chem. Rev. 113, 1686-1735. doi: $10.1021 / \mathrm{cr} 300047 \mathrm{q}$

Dykman, L., and Khlebtsov, N. (2012). Gold nanoparticles in biomedical applications: recent advances and perspectives. Chem. Soc. Rev. 41, 2256-2282. doi: 10.1039/C1CS15166E

Elsabahy, M., Heo, G. S., Lim, S. M., Sun, G. R., and Wooley, K. L. (2015). Polymeric nanostructures for imaging and therapy. Chem. Rev. 115, 10967-11011. doi: 10.1021/acs.chemrev.5b00135

Erogbogbo, F., Yong, K. T., Roy, I., Xu, G. X., Prasad, P. N., and Swihart, M. T. (2008). Biocompatible luminescent silicon quantum dots for imaging of cancer cells. ACS Nano 2, 873-878. doi: 10.1021/nn700319z

Fan, Q. L., Cheng, K., Yang, Z., Zhang, R. P., Yang, M., Hu, X., et al. (2015). Perylene-diimide-based nanoparticles as highly efficient photoacoustic agents for deep brain tumor imaging in living mice. Adv. Mater. 27, 843-847. doi: 10.1002/adma.201402972

Fan, W. P., Yung, B., Huang, P., and Chen, X. Y. (2017). Nanotechnology for multimodal synergistic cancer therapy. Chem. Rev. 117, 13566-13638. doi: 10.1021/acs.chemrev.7b00258

Farokhzad, O. C., and Langer, R. (2006). Nanomedicine: developing smarter therapeutic and diagnostic modalities. Adv. Drug Delivery Rev. 58, 1456-1459. doi: 10.1016/j.addr.2006.09.011

Feng, G., Fang, Y., Liu, J., Geng, J., Ding, D., and Liu, B. (2017). Multifunctional conjugated polymer nanoparticles for image-guided photodynamic and photothermal therapy. Small 13:1602807. doi: 10.1002/smll.201602807

Feng, L., Zhu, C., Yuan, H., Liu, L., Lv, F., and Wang, S. (2013). Conjugated polymer nanoparticles: preparation, properties, functionalization and biological applications. Chem. Soc. Rev. 42, 6620-6633. doi: 10.1039/c3cs60036j

Feng, T., Ai, X. Z., An, G. H., Yang, P. P., and Zhao, Y. L. (2016). Charge-convertible carbon dots for imaging guided drug delivery with enhanced in vivo cancer therapeutic efficiency. ACS Nano 10, 4410-4420. doi: 10.1021/acsnano.6b00043

Fu, X. Y., Liu, C. L., Shi, J. P., Man, H. Z., Xu, J., and Zhang, H. W. (2014). Long persistent near infrared luminescence nanoprobes

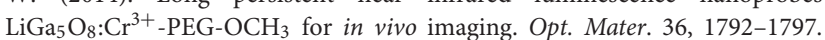
doi: 10.1016/j.optmat.2014.04.018

Gai, S. L., Yang, G. X., Yang, P. P., He, F., Lin, J., Jin, D. Y., et al. (2018). Recent advances in functional nanomaterials for light-triggered cancer therapy. Nano Today 19, 146-187. doi: 10.1016/j.nantod.2018.02.010

Gandra, N., and Singamaneni, S. (2013). Surface-enhanced Raman scattering for in vivo imaging: the future looks BRIGHT? Nanomedicine 8, 317-320. doi: $10.2217 / \mathrm{nnm} .13 .10$

Ge, J. C., Jia, Q. Y., Liu, W. M., Guo, L., Liu, Q. Y., Lan, M. H., et al. (2015). Redemissive carbon dots for fluorescent, photoacoustic, and thermal theranostics in living mice. Adv. Mater. 27, 4169-4177. doi: 10.1002/adma.201500323

Geng, J., Liao, L. D., Qin, W., Tang, B. Z., Thakor, N., and Liu, B. (2015). Fluorogens with aggregation induced emission: ideal photoacoustic contrast reagents due to intramolecular rotation. J. Nanosci. Nanotechnol. 15, 1864-1868. doi: 10.1166/jnn.2015.10031

Geszke-Moritz, M., and Moritz, M. (2013). Quantum dots as versatile probes in medical sciences: synthesis, modification and properties. Mat. Sci. Eng. Mater. 33, 1008-1021. doi: 10.1016/j.msec.2013.01.003

Goodarzi, S., Da Ros, T., Conde, J., Sefat, F., and Mozafari, M. (2017). Fullerene: biomedical engineers get to revisit an old friend. Mater. Today 20, 460-480. doi: 10.1016/j.mattod.2017.03.017

Grimaldi, N., Andrade, F., Segovia, N., Ferrer-Tasies, L., Sala, S., and Veciana, J. (2016) Lipid-based nanovesicles for nanomedicine. Chem. Soc. Rev. 45, 6520-6545. doi: 10.1039/C6CS00409A

Gu, B., Wu, W., Xu, G., Feng, G., Yin, F., Chong, P. H. J.,et al. (2017). Precise two-photon photodynamic therapy using an efficient photosensitizer with aggregation-induced emission characteristics. Adv. Mater. 29:1701076 doi: 10.1002/adma.201701076

Gu, L., Park, J. H., Duong, K. H., Ruoslahti, E., and Sailor, M. J. (2010). Magnetic luminescent porous silicon microparticles for localized delivery of molecular drug payloads. Small 6, 2546-2552. doi: 10.1002/smll.201000841

Gu, Z., Yan, L., Tian, G., Li, S., Chai, Z., and Zhao, Y. (2013). Recent advances in design and fabrication of upconversion nanoparticles and their safe theranostic applications. Adv. Mater. 25, 3758-3779. doi: 10.1002/adma. 201301197

Guerrini, L., Pazos-Perez, N., Garcia-Rico, E., and Alvarez-Puebla, R. (2017). Cancer characterization and diagnosis with SERS-encoded particles. Cancer Nanotechnol. 8:UNSP5. doi: 10.1186/s12645-017-0031-3

Guo, J., Rahme, K., He, Y., Li, L. L., Holmes, J. D., and O’Driscoll, C. M. (2017). Gold nanoparticles enlighten the future of cancer theranostics. Int. J. Nanomed. 12, 6131-6152. doi: 10.2147/IJN.S140772

Her, S., Jaffray, D. A., and Allen, C. (2017). Gold nanoparticles for applications in cancer radiotherapy: mechanisms and recent advancements. Adv. Drug Deliv. Rev. 109, 84-101. doi: 10.1016/j.addr.2015.12.012

Herranz-Blanco, B., Shahbazi, M. A., Correia, A. R., Balasubramanian, V., Kohout, T., Hirvonen, J., et al. (2016). pH-switch nanoprecipitation of polymeric nanoparticles for multimodal cancer targeting and intracellular triggered delivery of doxorubicin. Adv. Healthcare Mater. 5, 1904-1916. doi: 10.1002/adhm.201600160

Hessel, C. M., Pattani, V. P., Rasch, M., Panthani, M. G., Koo, B., Tunnell, J. W., et al. (2011). Copper selenide nanocrystals for photothermal therapy. Nano Lett. 11, 2560-2566. doi: 10.1021/nl201400z

Homan, K. A., Souza, M., Truby, R., Luke, G. P., Green, C., Vreeland, E., et al. (2012). Silver nanoplate contrast agents for in vivo molecular photoacoustic imaging. ACS Nano. 6, 641-650. doi: 10.1021/nn204100n

Homayoni, H., Ma, L., Zhang, J. Y., Sahi, S. K., Rashidi, L. H., Bui, B., et al. (2016). Synthesis and conjugation of $\mathrm{Sr}_{2} \mathrm{MgSi}_{2} \mathrm{O}_{7}: \mathrm{Eu}^{2+}, \mathrm{Dy}^{3+}$ water soluble afterglow nanoparticles for photodynamic activation. Photodiagn. Photodyn. Ther. 16, 90-99. doi: 10.1016/j.pdpdt.2016.08.012

Hong, G., Lee, J. C., Robinson, J. T., Raaz, U., Xie, L., Huang, N. F., et al. (2012a). Multifunctional in vivo vascular imaging using near-infrared II fluorescence. Nat. Med. 18, 1841-1846. doi: 10.1038/nm.2995

Hong, G., Robinson, J. T., Zhang, Y., Diao, S., Antaris, A. L., Wang, Q., et al. (2012b). in vivo fluorescence imaging with $\mathrm{Ag}_{2} \mathrm{~S}$ quantum dots in the second near-infrared region. Angew. Chem. Int. Ed. 51, 9818-9821. doi: 10.1002/anie.201206059

Hong, G. S., Diao, S. O., Antaris, A. L., and Dai, H. J. (2015). Carbon nanomaterials for biological imaging and nanomedicinal therapy. Chem. Rev. 115, 10816-10906. doi: 10.1021/acs.chemrev.5b00008

Hong, G. S., Zou, Y. P., Antaris, A. L., Diao, S., Wu, D., Cheng, K., et al. (2014). Ultrafast fluorescence imaging in vivo with conjugated polymer fluorophores in the second near-infrared window. Nat. Commun. 5:4206. doi: $10.1038 /$ ncomms5206

Hong, Y. N., Lam, J. M. Y., and Tang, B. Z. (2009). Aggregation-induced emission: phenomenon, mechanism and applications. Chem. Commun. 29, 4332-4353. doi: 10.1039/b904665h

Hong, Y. N., Lam, J. W. Y., and Tang, B. Z. (2011). Aggregation-induced emission. Chem. Soc. Rev. 40, 5361-5388. doi: 10.1039/c1cs15113d

$\mathrm{Hu}, \mathrm{F} ., \mathrm{Xu}, \mathrm{S}$., and Liu, B. (2018a). Photosensitizers with aggregation-induced emission: materials and biomedical applications. Adv. Mater. 30:1801350. doi: 10.1002/adma.201801350

Hu, L. D., Wang, P. Y., Zhao, M. Y., Liu, L., Zhou, L., Li, B. H., et al. (2018b). Near-infrared rechargeable "optical battery" implant for irradiation-free photodynamic therapy. Biomaterials 163, 154-162.doi: 10.1016/j.biomaterials.2018.02.029

Hu, R., Leung, N. L. C., and Tang, B. Z. (2014). AIE macromolecules: syntheses, structures and functionalities. Chem. Soc. Rev. 43, 4494-4562. doi: 10.1039/C4CS00044G

Huang, K., Li, Z. J., Lin, J., Han, G., and Huang, P. (2018). Two-dimensional transition metal carbides and nitrides (MXenes) for biomedical applications. Chem. Soc. Rev. 47, 5109-5124. doi: 10.1039/C7CS00838D

Huang, P., Rong, P. F., Jin, A., Yan, X. F., Zhang, M. G., Lin, J., et al. (2014b). Dyeloaded ferritin nanocages for multimodal imaging and photothermal therapy. Adv. Mater. 26, 6401-6408. doi: 10.1002/adma.201400914 
Huang, P., Rong, P. F., Lin, J., Li, W. W., Yan, X. F., Zhang, M. G., et al. (2014a). Triphase interface synthesis of plasmonic gold bellflowers as nearinfrared light mediated acoustic and thermal theranostics. J. Am. Chem. Soc. 136, 8307-8313.doi: 10.1021/ja503115n

Huang, X., and El-Sayed, M. A. (2010). Gold nanoparticles: optical properties and implementations in cancer diagnosis and photothermal therapy. J. Adv. Res. 1, 13-28. doi: 10.1016/j.jare.2010.02.002

Huang, Y. A., Xiao, Q. B., Hu, H. S., Zhang, K. C., Feng, Y. M., Li, F. J., et al. (2016). $915 \mathrm{~nm}$ light-triggered photodynamic therapy and MR/CT dualmodal imaging of tumor based on the nonstoichiometric $\mathrm{Na}_{0.52} \mathrm{YbF}_{3.52}$ : $\mathrm{Er}$ upconversion nanoprobes. Small 12, 4200-4210. doi: 10.1002/smll.201601023

Idris, N. M., Jayakumar, M. K. G., Bansal, A., and Zhang, Y. (2015). Upconversion nanoparticles as versatile light nanotransducers for photoactivation applications. Chem. Soc. Rev. 44, 1449-1478. doi: 10.1039/C4CS00158C

Jiang, K., Sun, S., Zhang, L., Lu, Y., Wu, A., and Cai, C. (2015). Red, green, and blue luminescence by carbon dots: full-color emission tuning and multicolor cellular imaging. Angew. Chem. Int. Ed. 54, 5360-5363. doi: 10.1002/anie.201501193

Jiang, Y., Upputuri, P. K., Xie, C., Zeng, Z., Sharma, A., Zhen, X., et al. (2019). Metabolizable semiconducting polymer nanoparticles for second near-infrared photoacoustic imaging. Adv. Mater. 31:1808166. doi: 10.1002/adma.201808166

Jung, H. S., Verwilst, P., Sharma, A., Shin, J., Sessler, J. L., and Kim, J. S. (2018). Organic molecule-based photothermal agents: an expanding photothermal therapy universe. Chem. Soc. Rev. 47, 2280-2297. doi: 10.1039/C7CS00522A

Kamimura, S., Xu, C. N., Yamada, H., Marriott, G., Hyodo, K., and Ohno, T. (2017). Near-infrared luminescence from double-perovskite $\mathrm{Sr}_{3} \mathrm{Sn}_{2} \mathrm{O}_{7}: \mathrm{Nd}^{3+}$ : a new class of probe for in vivo imaging in the second optical window of biological tissue. J. Ceram. Soc. Jpn. 125, 591-595. doi: 10.2109/jcersj2.17051

Kamimura, S., Xu, C. N., Yamada, H., Terasaki, N., and Fujihala, M. (2014). Long-persistent luminescence in the near-infrared from $\mathrm{Nd}^{3+}$. doped $\mathrm{Sr}_{2} \mathrm{SnO}_{4}$ for in vivo optical imaging. Jpn. J. Appl. Phys. 53:092403. doi: $10.7567 /$ JJAP.53.092403

Karimi, M., Ghasemi, A., Zangabad, P. S., Rahighi, R., Basri, S. M. M., Mirshekari, H., et al. (2016). Smart micro/nanoparticles in stimulus-responsive drug/gene delivery systems. Chem. Soc. Rev. 45, 1457-1501. doi: 10.1039/C5CS00798D

Kharlamov, A. N., Tyurnina, A. E., Veselova, V. S., Kovtun, O. P., Shur, V. Y., and Gabinsky, J. L. (2015). Silica-gold nanoparticles for atheroprotective management of plaques: results of the NANOM-FIM trial. Nanoscale 7, 8003-8015. doi: 10.1039/C5NR01050K

Kim, C., Favazza, C., and Wang, L. H. V. (2010). in vivo photoacoustic tomography of chemicals: high-resolution functional and molecular optical imaging at new depths. Chem. Rev. 110, 2756-2782. doi: 10.1021/cr900266s

Krasia-Christoforou, T., and Georgiou, T. K. (2013). Polymeric theranostics: using polymer-based systems for simultaneous imaging and therapy. J. Mater. Chem. 1, 3002-3025. doi: 10.1039/c3tb20191k

Kumar, S., Sarita, N. M., Dilbaghi, N., and Tankeshwar, K., Kim, K. H. (2018). Recent advances and remaining challenges for polymeric nanocomposites in healthcare applications. Prog. Polym. Sci. 80, 1-38. doi: 10.1016/j.progpolymsci.2018.03.001

Kunjachan, S., Ehling, J., Storm, G., Kiessling, F., and Lammers, T. (2015). Noninvasive imaging of nanomedicines and nanotheranostics: principles, progress, and prospects. Chem. Rev. 115, 10907-10937. doi: 10.1021/cr500314d

Lalwani, G., Cai, X., Nie, L. M., Wang, L. H. V., and Sitharaman, B. (2013). Graphene-based contrast agents for photoacoustic and thermoacoustic tomography. Photoacoustics 1, 62-67. doi: 10.1016/j.pacs.2013.10.001

Lamch, L., Pucek, A., Kulbacka, J., Chudy, M., Jastrzebska, E., Tokarska, K., et al. (2018). Recent progress in the engineering of multifunctional colloidal nanoparticles for enhanced photodynamic therapy and bioimaging. $A d v$. Colloid. Interfaces 261, 62-81. doi: 10.1016/j.cis.2018.09.002

Lan, M., Zhao, S., Liu, W., Lee, C. S., Zhang, W., and Wang, P. (2019). Photosensitizers for photodynamic therapy. Adv. Healthc. Mater. 8:e1900132. doi: 10.1002/adhm.201900132

Lee, C., Kwon, W., Beack, S., Lee, D., Park, Y., Kim, H., et al. (2016). Biodegradable nitrogen-doped carbon nanodots for non-invasive photoacoustic imaging and photothermal therapy. Theranostics 6, 2196-2208. doi: 10.7150/thno.16923

Lee, D. E., Koo, H., Sun, I. C., Ryu, J. H., Kim, K., and Kwon, I. C. (2012). Multifunctional nanoparticles for multimodal imaging and theragnosis. Chem. Soc. Rev. 41, 2656-2672. doi: 10.1039/C2CS15261D
Li, H., Kang, Z., Liu, Y., and Lee, S. T. (2012). Carbon nanodots: synthesis, properties and applications. J. Mater. Chem. 22, 24230-24253. doi: $10.1039 / \mathrm{c} 2 \mathrm{jm} 34690 \mathrm{~g}$

Li, J., and Pu, K. (2019). Development of organic semiconducting materials for deep-tissue optical imaging, phototherapy and photoactivation. Chem. Soc. Rev. 48, 38-71. doi: 10.1039/C8CS00001H

Li, J., Xie, C., Huang, J., Jiang, Y., Miao, Q., and Pu, K. (2018d). Semiconducting polymer nanoenzymes with photothermic activity for enhanced cancer therapy. Angew. Chem. Int. Ed. 130, 4059-4062. doi: 10.1002/ange.201800511

Li, J. L., Shi, J. P., Shen, J. S., Man, H. Z., Wang, M. X., and Zhang, H. W. (2015b). Specific recognition of breast cancer cells in vitro using near infrared-emitting long-persistence luminescent $\mathrm{Zn}_{3} \mathrm{Ga}_{2} \mathrm{Ge}_{2} \mathrm{O}_{10}$ : $\mathrm{Cr}^{3+}$ nanoprobes. Nano-Micro Lett. 7, 138-145. doi: 10.1007/s40820-014-0026-0

Li, J. L., Shi, J. P., Wang, C. C., Li, P. H., Yu, Z. F., and Zhang, H. W. (2017a). Five-nanometer $\mathrm{ZnSn}_{2} \mathrm{O}_{4}$ : $\mathrm{Cr}$, Eu ultra-small nanoparticles as new near infrared-emitting persistent luminescent nanoprobes for cellular and deep tissue imaging at $800 \mathrm{~nm}$. Nanoscale 9, 8631-8638. doi: 10.1039/C7NR02468A

Li, J. L., Wang, C. C., Shi, J. P., Li, P. H., Yu, Z. F., and Zhang, H. W. (2018b). Porous $\mathrm{GdAlO}_{3}: \mathrm{Cr}^{3+}, \mathrm{Sm}^{3+}$ drug carrier for real-time long afterglow and magnetic resonance dual-mode imaging. J. Lumin. 199, 363-371. doi: 10.1016/j.jlumin.2018.03.071

Li, S. L., Wang, X. Y., Hu, R., Chen, H., Li, M., Wang, J. W., et al. (2016). Near-infrared (NIR)-absorbing conjugated polymer dots as highly effective photothermal materials for in vivo cancer therapy. Chem. Mater. 28, 8669-8675. doi: 10.1021/acs.chemmater.6b03738

Li, W., and Chen, X. Y. (2015). Gold nanoparticles for photoacoustic imaging. Nanomedicine 10, 299-320. doi: 10.2217/nnm.14.169

Li, W., Liu, Z. H., Fontana, F., Ding, Y. P., Liu, D. F., Hirvonen, J. T., et al. (2018a). Tailoring porous silicon for biomedical applications: from drug delivery to cancer immunotherapy. Adv. Mater. 30:1703740. doi: 10.1002/adma.201703740

Li, W., Zhang, Z., Kong, B., Feng, S., Wang, J., Wang, L., et al. (2013). Simple and green synthesis of nitrogen-doped photoluminescent carbonaceous nanospheres for bioimaging. Angew. Chem. Int. Ed. 52, 8151-8155. doi: 10.1002/anie.201303927

Li, X., Shan, J. Y., Zhang, W. Z., Su, S., Yuwen, L. H., and Wang, L. H. (2017b). Recent advances in synthesis and biomedical applications of twodimensional transition metal sichalcogenide nanosheets. Small 13:1602660. doi: 10.1002/smll.201602660

Li, Y., Zhou, S. F., Dong, G. P., Peng, M. Y., Wondraczek, L., and Qiu, J. R. (2014a). Anti-stokes fluorescent probe with incoherent excitation. Sci. Rep. 4:4059. doi: 10.1038/srep04059

Li, Y., Zhou, S. F., Li, Y. Y., Sharafudeen, K., Ma, Z. J., Dong, G. P., et al. (2014b). Long persistent and photo-stimulated luminescence in $\mathrm{Cr}^{3+}$-doped $\mathrm{Zn}$-GaSn-O phosphors for deep and reproducible tissue imaging. J. Mater. Chem. 2, 2657-2663. doi: 10.1039/c4tc00014e

Li, Y. J., and Yan, X. P. (2016). Synthesis of functionalized triple-doped zinc gallogermanate nanoparticles with superlong near-infrared persistent luminescence for long-term orally administrated bioimaging. Nanoscale 8, 14965-14970. doi: 10.1039/C6NR04950H

Li, Y. J., Yang, C. X., and Yan, X. P. (2018c). Biomimetic persistent luminescent nanoplatform for autofluorescence-free metastasis tracking and chemophotodynamic therapy. Anal. Chem. 90, 4188-4195. doi: 10.1021/acs.analchem.8b00311

Li, Z., and Yang, Y. W. (2017). Creation and bioapplications of porous organic polymer materials. J. Mater. Chem. 5, 9278-9290. doi: 10.1039/C7TB02647A

Li, Z., Zhang, Y., Wu, X., Huang, L., Li, D., Fan, W., et al. (2015a) Direct aqueous-phase synthesis of sub-10 nm "luminous pearls" with enhanced in vivo renewable near-infrared persistent luminescence. J. Am. Chem. Soc. 137, 5304-5307. doi: 10.1021/jacs.5b00872

Li, Z. J., Shi, J. P., Zhang, H. W., and Sun, M. (2014c) Highly controllable synthesis of near-infrared persistent luminescence $\mathrm{SiO}_{2} / \mathrm{CaMgSi}_{2} \mathrm{O}_{6}$ composite nanospheres for imaging in vivo. Opt. Exp. 22, 10509-10518. doi: 10.1364/OE.22.010509

Liang, C., Xu, L. G., Song, G. S., and Liu, Z. (2016). Emerging nanomedicine approaches fighting tumor metastasis: animal models, metastasis-targeted drug delivery, phototherapy, and immunotherapy. Chem. Soc. Rev. 45, 6250-6269. doi: 10.1039/C6CS00458J 
Lim, E. K., Kim, T., Paik, S., Haam, S., Huh, Y. M., and Lee, K. (2015). Nanomaterials for theranostics: recent advances and future challenges. Chem. Rev. 115, 327-394. doi: 10.1021/cr300213b

Lin, L. S., Cong, Z. X., Cao, J. B., Ke, K. M., Peng, Q. L., Gao, J. H., et al. (2014). Multifunctional $\mathrm{Fe}_{3} \mathrm{O}_{4} @$ polydopamine core-shell nanocomposites for intracellular mRNA detection and imaging-guided photothermal therapy. ACS Nano 8, 3876-3883. doi: 10.1021/nn500722y

Lin, W. H., Zhang, W., Sun, T. T., Liu, S., Zhu, Y., and Xie, Z. G. (2017). Rational design of polymeric nanoparticles with tailorable biomedical functions for cancer. ACS Appl. Mater. Interfac. 9, 29612-29622. doi: 10.1021/acsami.7b10763

Liu, F., Liang, Y. J., and Pan, Z. W. (2014b). Detection of upconverted persistent luminescence in the near infrared emitted by the $\mathrm{Zn}_{3} \mathrm{Ga}_{2} \mathrm{GeO}_{8}: \mathrm{Cr}^{3+}, \mathrm{Yb}^{3+}, \mathrm{Er}^{3+}$ phosphor. Phys. Rev. Lett. 113:177401. doi: 10.1103/PhysRevLett.113.177401

Liu, F., Yan, W. Z., Chuang, Y. J., Zhen, Z. P., Xie, J., and Pan, Z. W. (2013). Photostimulated near-infrared persistent luminescence as a new optical readout from $\mathrm{Cr}^{3+}$-doped $\mathrm{LiGa}_{5} \mathrm{O}_{8}$. Sci. Rep. 3:1554. doi: 10.1038/srep01554

Liu, F. Y., He, X. X., Chen, H. D., Zhang, J. P., Zhang, H. M., and Wang, Z. X. (2015c). Gram-scale synthesis of coordination polymer nanodots with renal clearance properties for cancer theranostic applications. Nat. Commun. 6:8003. doi: $10.1038 /$ ncomms 9003

Liu, H. H., Ren, F., Zhang, H., Han, Y. B., Qin, H. Z., Zeng, J. F., et al. (2018a). Oral administration of highly bright $\mathrm{Cr}^{3+}$ doped $\mathrm{ZnGa}_{2} \mathrm{O}_{4}$ nanocrystals for in vivo targeted imaging of orthotopic breast cancer. J. Mater. Chem. 6, 1508-1518. doi: 10.1039/C7TB03148C

Liu, J., Zheng, X. P., Yan, L., Zhou, L. J., Tian, G., Yin, W. Y., et al. (2015e). Bismuth sulfide nanorods as a precision nanomedicine for in vivo multimodal imaging-guided photothermal therapy of tumor. ACS Nano 9, 696-707. doi: $10.1021 / \mathrm{nn} 506137 \mathrm{n}$

Liu, J. H., Cao, L., LeCroy, G. E., Wang, P., Meziani, M. J., Dong, Y. Y., et al. (2015a). Carbon "quantum" dots for fluorescence labeling of cells. ACS Appl. Mater. Interfaces 7, 19439-19445. doi: 10.1021/acsami.5b05665

Liu, J. H., Lecuyer, T., Seguin, J., Mignet, N., Scherman, D., Viana, B., et al. (2019a). Imaging and therapeutic applications of persistent luminescence nanomaterials. Adv. Drug Delivery Rev. 138, 193-210. doi: 10.1016/j.addr.2018.10.015

Liu, J. M., Liu, Y. Y., Zhang, D. D., Fang, G. Z., and Wang, S. (2016). Synthesis of $\mathrm{GdAlO}_{3}: \mathrm{Mn}^{4+}, \mathrm{Ge}^{4+} \mathrm{Au}$ core-shell nanoprobes with plasmon-enhanced nearinfrared persistent luminescence for in vivo trimodality bioimaging. ACS Appl. Mater. Interfaces 8, 29939-29949. doi: 10.1021/acsami.6b09580

Liu, J. M., Zhang, D. D., Fang, G. Z., and Wang, S. (2018b). Erythrocyte membrane bioinspired near-infrared persistent luminescence nanocarriers for in vivo long-circulating bioimaging and drug delivery. Biomaterials 165, 39-47. doi: 10.1016/j.biomaterials.2018.02.042

Liu, Q., Feng, W., and Li, F. Y. (2014a). Water-soluble lanthanide upconversion nanophosphors: synthesis and bioimaging applications in vivo. Coord. Chem. Rev. 273, 100-110. doi: 10.1016/j.ccr.2014.01.004

Liu, X. G., Yan, C. H., and Capobianco, J. A. (2015b). Photon upconversion nanomaterials. Chem. Soc. Rev. 44, 1299-1301. doi: 10.1039/C5CS90009C

Liu, Y. J., Bhattarai, P., Dai, Z. F., and Chen, X. Y. (2019b). Photothermal therapy and photoacoustic imaging via nanotheranostics in fighting cancer. Chem. Soc. Rev. 48, 2053-2108. doi: 10.1039/C8CS00618K

Liu, Y. Y., Liu, J. M., Zhang, D. D., Ge, K., Wang, P. H., Liu, H. L., et al. (2017). Persistent luminescence nanophosphor involved near-infrared optical bioimaging for investigation of foodborne probiotics biodistribution in vivo: a proof-of-concept study. J. Agr. Food Chem. 65, 8229-8240. doi: 10.1021/acs.jafc.7b02870

Liu, Z., Robinson, J. T., Tabakman, S. M., Yang, K., and Dai, H. J. (2011). Carbon materials for drug delivery \& cancer therapy. Mater. Today 14, 316-323. doi: 10.1016/S1369-7021(11)70161-4

Liu, Z. M., Ye, B. G., Jin, M., Chen, H. L., Zhong, H. Q., Wang, X. P., et al. (2015d). Dye-free near-infrared surface-enhanced Raman scattering nanoprobes for bioimaging and high-performance photothermal cancer therapy. Nanoscale 7, 6754-6761. doi: 10.1039/C5NR 01055A

Loni, A., Defforge, T., Caffull, E., Gautier, G., and Canham, L. T. (2015). Porous silicon fabrication by ariodisation: progress towards the realisation of layers and powders with high surface area and micropore content. Micropor. Mesopor. Mater. 213, 188-191. doi: 10.1016/j.micromeso.2015.03.006

Lovell, J. F., Jin, C. S., Huynh, E., Jin, H. L., Kim, C., Rubinstein, J. L., et al. (2011). Porphysome nanovesicles generated by porphyrin bilayers for use as multimodal biophotonic contrast agents. Nat. Mater. 10, 324-332. doi: 10.1038/nmat2986

Lu, D., Tao, R., and Wang, Z. (2019). Carbon-based materials for photodynamic therapy: a mini-review. Front. Chem. Sci. Eng. 13, 310-323. doi: $10.1007 / \mathrm{s} 11705-018-1750-7$

Lu, Z. S., and Li, C. M. (2011). Quantum dot-based nanocomposites for biomedical applications. Curr. Med. Chem. 18, 3516-3528. doi: 10.2174/092986711796642634

Lucky, S. S., Soo, K. C., and Zhang, Y. (2015). Nanoparticles in photodynamic therapy. Chem. Rev. 115, 1990-2042. doi: 10.1021/cr5004198

Luk, B. T., and Zhang, L. F. (2014). Current advances in polymer-based nanotheranostics for cancer treatment and diagnosis. ACS Appl. Mater. Interfaces 6, 21859-21873. doi: 10.1021/am5036225

Luo, J. D., Xie, Z. L., Lam, J. W. Y., Cheng, L., Chen, H. Y., Qiu, C. F., et al. (2001). Aggregation-induced emission of 1-methyl-1,2,3,4,5-pentaphenylsilole. Chem. Commun. 18, 1740-1741. doi: 10.1039/b105159h

Lyu, Y., Fang, Y., Miao, Q. Q., Zhen, X., Ding, D., and Pu, K. Y. (2016). Intraparticle molecular orbital engineering of semiconducting polymer nanoparticles as amplified theranostics for in vivo photoacoustic imaging and photothermal therapy. ACS Nano 10, 4472-4481. doi: 10.1021/acsnano.6b00168

Lyu, Y., and Pu, K. (2017). Recent advances of activatable molecular probes based on semiconducting polymer nanoparticles in sensing and imaging. Adv. Sci. 4:1600481. doi: 10.1002/advs.201600481

Lyu, Y., Zeng, J., Jiang, Y., Zhen, X., Wang, T., Qiu, S., et al. (2018). Enhancing both biodegradability and efficacy of semiconducting polymer nanoparticles for photoacoustic imaging and photothermal therapy. ACS Nano 12, 1801-1810. doi: 10.1021/acsnano.7b08616

Ma, P., and Mumper, R. J. (2013). Paclitaxel nano-delivery systems: a comprehensive review. J. Nanomed. Nanotechnol. 4:1000164. doi: $10.4172 / 2157-7439.1000164$

Maiti, K. K., Dinish, U. S., Samanta, A., Vendrell, M., Soh, K. S., Park, S. J., et al. (2012). Multiplex targeted in vivo cancer detection using sensitive near-infrared SERS nanotags. Nano Today 7, 85-93. doi: 10.1016/j.nantod.2012.02.008

Makila, E., Bimbo, L. M., Kaasalainen, M., Herranz, B., Airaksinen, A. J., Heinonen, M., et al. (2012). Amine modification of thermally carbonized porous silicon with silane coupling chemistry. Langmuir 28, 14045-14054. doi: 10.1021/la303091k

Maldiney, T., Ballet, B., Bessodes, M., Scherman, D., and Richard, C. (2014b). Mesoporous persistent nanophosphors for in vivo optical bioimaging and drug-delivery. Nanoscale 6, 13970-13976. doi: 10.1039/C4NR03843F

Maldiney, T., Bessiere, A., Seguin, J., Teston, E., Sharma, S. K., Viana, B., et al. (2014a). The in vivo activation of persistent nanophosphors for the optical imaging of vascularization, tumors and grafted cells. Nat. Mater. 13, 418-426. doi: $10.1038 /$ nmat 3908

Maldiney, T., Doan, B. T., Alloyeau, D., Bessodes, M., Scherman, D., and Richard, C. (2015). Gadolinium-doped persistent nanophosphors as versatile tool for multimodal in vivo imaging, Adv. Funct. Mater. 25, 331-338. doi: $10.1002 /$ adfm. 201401612

Maldiney, T., Lecointre, A., Viana, B., Bessiere, A., Bessodes, M., Gourier, D., et al. (2011). Controlling electron trap depth to enhance optical properties of persistent luminescence nanoparticles for in vivo imaging. J. Am. Chem. Soc. 133, 11810-11815. doi: 10.1021/ja204504w

Maldiney, T., Sraiki, G., Viana, B., Gourier, D., Richard, C., Scherman, D., et al. (2012). in vivo optical imaging with rare earth doped $\mathrm{Ca}_{2} \mathrm{Si}_{5} \mathrm{~N}_{8}$ persistent luminescence nanoparticles. Opt. Mater. Exp. 2, 261-268. doi: 10.1364/OME.2.000261

Mao, H. Y., Laurent, S., Chen, W., Akhavan, O., Imani, M., Ashkarran, A. A., et al. (2013). Graphene: promises, facts, opportunities, and challenges in nanomedicine. Chem. Rev. 113, 3407-3424. doi: 10.1021/cr300335p

Mei, J., Hong, Y. N., Lam, J. M. Y., Qin, A. J., Tang, Y. H., and Tang, B. Z. (2014). Aggregation-induced emission: the whole is more brilliant than the parts. $A d v$. Mater. 26, 5429-5479. doi: 10.1002/adma.201401356

Mei, J., Leung, N. L. C., Kwok, R. T. K., Lam, J. W. Y., and Tang, B. Z. (2015). Aggregation-induced emission: together we shine, united 
we soar! Chem. Rev. 115, 11718-11940. doi: 10.1021/acs.chemrev.5 b00263

Miao, Q. Q., and Pu, K. Y. (2018). Organic semiconducting agents for deep-tissue molecular imaging: second near-infrared fluorescence, self-luminescence, and photoacoustics. Adv. Mater. 30:1801778. doi: 10.1002/adma.201801778

Min, Y. Z., Li, J. M., Liu, F., Yeow, E. K. L., and Xing, B. G. (2014). Near-infrared light-mediated photoactivation of a platinum antitumor prodrug and simultaneous cellular apoptosis imaging by upconversionluminescent nanoparticles. Angew. Chem. Int. Ed. 53, 1012-1016. doi: 10.1002/anie.201308834

Mishra, V., Patil, A., Thakur, S., and Kesharwani, P. (2018). Carbon dots: emerging theranostic nanoarchitectures. Drug Discov. Today 23, 1219-1232. doi: 10.1016/j.drudis.2018.01.006

Mo, D., Hu, L., Zeng, G. M., Chen, G. Q., Wan, J., Yu, Z. G., et al. (2017). Cadmium-containing quantum dots: properties, applications, and toxicity. Appl. Microbiol. Biotechnol. 101, 2713-2733. doi: 10.1007/s00253-017-8140-9

Mochalin, V. N., Pentecost, A., Li, X. M., Neitzel, I., Nelson, M., Wei, C. Y., et al. (2013). Adsorption of drugs on nanodiamond: toward development of a drug delivery platform. Mol. Pharmaceut. 10, 3728-3735. doi: 10.1021/mp400213z

Mochalin, V. N., Shenderova, O., Ho, D., and Gogotsi, Y. (2011). The properties and applications of nanodiamonds. Nat. Nanotechnol. 7, 11-23. doi: 10.1038/nnano.2011.209

Montalti, M., Prodi, L., Rampazzo, E., and Zaccheroni, N. (2014). Dye-doped silica nanoparticles as luminescent organized systems for nanomedicine. Chem. Soc. Rev. 43, 4243-4268. doi: 10.1039/C3CS60433K

Namdari, P., Negahdari, B., and Eatemadi, A. (2017). Synthesis, properties and biomedical applications of carbon-based quantum dots: an updated review. Biomed. Pharmacother. 87, 209-222. doi: 10.1016/j.biopha.2016.12.108

Nicolas, J., Mura, S., Brambilla, D., Mackiewicz, N., and Couvreur, P. (2013). Design, functionalization strategies and biomedical applications of targeted biodegradable/biocompatible polymer-based nanocarriers for drug delivery. Chem. Soc. Rev. 42, 1147-1235. doi: 10.1039/C2CS35265F

Nie, L. M., Chen, M., Sun, X. L., Rong, P. F., Zheng, N. F., and Chen, X. Y. (2014). Palladium nanosheets as highly stable and effective contrast agents for in vivo photoacoustic molecular imaging. Nanoscale 6, 1271-1276. doi: $10.1039 / \mathrm{C} 3 \mathrm{NR} 05468 \mathrm{C}$

Nie, L. M., and Chen, X. Y. (2014). Structural and functional photoacoustic molecular tomography aided by emerging contrast agents. Chem. Soc. Rev. 43, 7132-7170. doi: 10.1039/C4CS00086B

Ntziachristos, V. (2010). Going deeper than microscopy: the optical imaging frontier in biology. Nat. Methods 7, 603-614. doi: 10.1038/nmeth.1483

Panwar, N., Soehartono, A. M., Chan, K. K., Zeng, S., Xu, G., Qu, J., et al. (2019). Nanocarbons for biology and medicine: sensing, imaging, and drug delivery. Chem. Rev. 119, 9559-9656. doi: 10.1021/acs.chemrev.9b00099

Park, J. H., Gu, L., von Maltzahn, G., Ruoslahti, E., Bhatia, S. N., and Sailor, M. J. (2009). Biodegradable luminescent porous silicon nanoparticles for in vivo applications. Nat. Mater. 8, 331-336. doi: 10.1038/nmat2398

Patel, K. D., Singh, R. K., and Kim, H. W. (2019). Carbon-based nanomaterials as an emerging platform for theranostics. Mater. Horiz. 6, 434-469. doi: 10.1039/C8MH00966 J

Pecher, J., and Mecking, S. (2010). Nanoparticles of conjugated polymers. Chem. Rev. 110, 6260-6279. doi: 10.1021/cr100132y

Perrin, L., and Hudhomme, P. (2011). Synthesis, electrochemical and optical absorption properties of new perylene-3,4:9,10-bis(dicarboximide) and perylene-3,4:9,10-bis(benzimidazole) derivatives. Eur. J. Org. Chem. 2011, 5427-5440. doi: 10.1002/ejoc.201100513

Pichaandi, J., and van Veggel, F. C. J. M. (2014). Near-infrared emitting quantum dots: recent progress on their synthesis and characterization. Coord. Chem. Rev. 263, 138-150. doi: 10.1016/j.ccr.2013.10.011

Premasiri, W. R., Chen, Y., Fore, J., Brodeur, A., and Ziegler, L. D. (2018). "Chapter 10 - SERS biomedical applications: diagnostics, forensics, and metabolomics," in Frontiers and Advances in Molecular Spectroscopy, ed J. Laane (Cambridge, MA: Elsevier Press), 327-367. doi: 10.1016/B978-0-12-811220-5.00010-1

Probst, C. E., Zrazhevskiy, P., Bagalkot, V., and Gao, X. H. (2013). Quantum dots as a platform for nanoparticle drug delivery vehicle design. Adv. Drug Deliv. Rev. 65, 703-718. doi: 10.1016/j.addr.2012.09.036

Pu, K., Mei, J. G., Jokerst, J. V., Hong, G. S., Antaris, A. L., Chattopadhyay, N., et al. (2015). Diketopyrrolopyrrole-based semiconducting polymer nanoparticles for in vivo photoacoustic imaging. Adv. Mater. 27, 5184-5190. doi: 10.1002/adma.201502285

Pu, K. Y., Chattopadhyay, N., and Rao, J. H. (2016). Recent advances of semiconducting polymer nanoparticles in in vivo molecular imaging. J. Control. Release 240, 312-322. doi: 10.1016/j.jconrel.2016.01.004

Pu, K. Y., Shuhendler, A. J., Jokerst, J. V., Mei, J. G., Gambhir, S. S., Bao, Z. N., et al. (2014a). Semiconducting polymer nanoparticles as photoacoustic molecular imaging probes in living mice. Nat. Nanotechnol. 9, 233-239. doi: 10.1038/nnano.2013.302

Pu, K. Y., Shuhendler, A. J., Valta, M. P., Cui, L. N., Saar, M., Peehl, D. M., et al. (2014b). Phosphorylcholine-coated semiconducting polymer nanoparticles as rapid and efficient labeling agents for in vivo cell tracking. Adv. Healthcare Mater. 3, 1292-1298. doi: 10.1002/adhm.201300534

Qiu, M., Singh, A., Wang, D., Qu, J. L., Swihart, M., Zhang, H., et al. (2019). Biocompatible and biodegradable inorganic nanostructures for nanomedicine: silicon and black phosphorus. Nano Today 25, 135-155. doi: 10.1016/j.nantod.2019.02.012

Ramirez-Garcia, G., Gutierrez-Granados, S., Gallegos-Corona, M. A., PalmaTirado, L., d'Orlye, F., Varenne, A., et al. (2017). Long-term toxicological effects of persistent luminescence nanoparticles after intravenous injection in mice. Int. J. Pharmaceut. 532, 686-695. doi: 10.1016/j.ijpharm.2017.07.015

Reina, G., Gonzalez-Dominguez, J. M., Criado, A., Vazquez, E., Bianco, A., and Prato, M. (2017). Promises, facts and challenges for graphene in biomedical applications. Chem. Soc. Rev. 46, 4400-4416. doi: 10.1039/C7CS00363C

Riley, R. S., and Day, E. S. (2017). Gold nanoparticle-mediated photothermal therapy: applications and opportunities for multimodal cancer treatment. Wiley Interdiscip. Rev. 9:e1449. doi: 10.1002/wnan.1449

Rong, P., Yang, K., Srivastan, A., Kiesewetter, D. O., Yue, X., Wang, F., et al. (2014). Photosensitizer loaded nano-graphene for multimodality imaging guided tumor photodynamic therapy. Theranostics 4, 229-239. doi: 10.7150/thno.8070

Rong, P. F., Huang, P., Liu, Z. G., Lin, J., Jin, A., Ma, Y., et al. (2015). Proteinbased photothermal theranostics for imaging-guided cancer therapy. Nanoscale 7, 16330-16336. doi: 10.1039/C5NR04428F

Rosticher, C., Chaneac, C., Viana, B., Fortin, M. A., Lagueux, J., and Faucher, L. (2015). Red persistent luminescence and magnetic properties of nanomaterials for multimodal imaging. Oxide-Based Mater. 9364:936419. doi: $10.1117 / 12.2087319$

Ryu, T. K., Baek, S. W., Kang, R. H., and Choi, S. W. (2016). Selective photothermal tumor therapy using nanodiamond-based nanoclusters with folic acid. $A d v$. Funct. Mater. 26, 6428-6436. doi: 10.1002/adfm.201601207

Sadegh, H., and Shahryari-ghoshekandi, R. (2015). Functionalization of carbon nanotubes and its application in nanomedicine: a review. Nanomed. J. 2, 231-248. doi: 10.7508/NMJ.2015.04.001

Sailor, M. J. (2012). Porous Silicon in Practice: Preparation, Characterization and Applications. Weinheim: Wiley-VCH Verlag GmbH \& Co. KGaA.

Shanmugam, V., Selvakumar, S., Yeh, C. S. (2014). Near-infrared light-responsive nanomaterials in cancer therapeutics. Chem. Soc. Rev. 43, 6254-6287. doi: 10.1039/C4CS00011K

Sheng, Z. H., Hu, D. H., Zheng, M. B., Zhao, P. F., Liu, H. L., Gao, D. Y., et al. (2014). Smart human serum albumin-indocyanine green nanoparticles generated by programmed assembly for dual-modal imaging-guided cancer synergistic phototherapy. ACS Nano 8, 12310-12322. doi: 10.1021/ nn5062386

Sheng, Z. H., Song, L., Zheng, J. X., Hu, D. H., He, M., Zheng, M. B., et al. (2013). Protein-assisted fabrication of nano-reduced graphene oxide for combined in vivo photoacoustic imaging and photothermal therapy. Biomaterials 34, 5236-5243. doi: 10.1016/j.biomaterials.2013.03.090

Shi, J. P., Sun, X., Li, J. L., Man, H. Z., Shen, J. S., Yu, Y. K., et al. (2015a). Multifunctional near infrared-emitting long-persistence luminescent nanoprobes for drug delivery and targeted tumor imaging. Biomaterials 37, 260-270. doi: 10.1016/j.biomaterials.2014.10.033

Shi, J. P., Sun, X., Zheng, S. H., Li, J. L., Fu, X. Y., Zhang, H. W. (2018). A new near-infrared persistent luminescence nanoparticle as a multifunctional nanoplatform for multimodal imaging and cancer therapy. Biomaterials 152, 15-23. doi: 10.1016/j.biomaterials.2017.10.032

Shi, S., Chen, F., Ehlerding, E. B., Cai, W. (2014). Surface engineering of graphenebased nanomaterials for biomedical applications. Bioconjugate Chem. 25, 1609-1619. doi: 10.1021/bc500332c 
Shi, S., Yang, K., Hong, H., Chen, F., Valdovinos, H. F., Goel, S., et al. (2015b). VEGFR targeting leads to significantly enhanced tumor uptake of nanographene oxide in vivo. Biomaterials 39, 39-46. doi: 10.1016/j.biomaterials.2014.10.061

Shuhendler, A. J., Pu, K. Y., Cui, L., Uetrecht, J. P., Rao, J. H. (2014). Real-time imaging of oxidative and nitrosative stress in the liver of live animals for drug-toxicity testing. Nat. Biotechnol. 32, 373-U240. doi: 10.1038/nbt.2838

Skrabalak, S. E., Chen, J., Sun, Y., Lu, X., Au, L., Cobley, C. M., et al. (2008). Gold nanocages: synthesis, properties, and applications. Acc. Chem. Res. 41, 1587-1595. doi: $10.1021 / \mathrm{ar} 800018 \mathrm{v}$

Smith, B. R., and Gambhir, S. S. (2017). Nanomaterials for in vivo imaging. Chem. Rev. 117, 901-986. doi: 10.1021/acs.chemrev.6b00073

Song, J. B., Yang, X. Y., Jacobson, O., Huang, P., Sun, X. L., Lin, L. S., et al. (2015a). Ultrasmall gold nanorod vesicles with enhanced tumor accumulation and fast excretion from the body for cancer therapy. Adv. Mater. 27, 4910-4917. doi: 10.1002/adma.201502486

Song, X. J., Chen, Q., Liu, Z. (2015c). Recent advances in the development of organic photothermal nano-agents. Nano Res. 8, 340-354. doi: $10.1007 / \mathrm{s} 12274-014-0620-y$

Song, X. R., Wang, X. Y., Yu, S. X., Cao, J. B., Li, S. H., Li, J., et al. (2015b). $\mathrm{Co}_{9} \mathrm{Se}_{8}$ nanoplates as a new theranostic platform for photoacoustic/magnetic resonance dual-modal-imaging-guided chemo-photothermal combination therapy. Adv. Mater. 27, 3285-3291. doi: 10.1002/adma.201405634

Spyratou, E., Makropoulou, M., Efstathopoulos, E. P., Georgakilas, A. G., Sihver, L. (2017). Recent advances in cancer therapy based on dual mode gold nanoparticles. Cancers 9:173. doi: 10.3390/cancers9120173

Sun, S. K., Wang, H. F., Yan, X. P. (2018). Engineering persistent luminescence nanoparticles for biological applications: from biosensing/bioimaging to theranostics. Accounts Chem. Res. 51, 1131-1143. doi: $10.1021 /$ acs.accounts.7b00619

Tan, C. L., Cao, X. H., Wu, X. J., He, Q. Y., Yang, J., Zhang, X., et al. (2017). Recent advances in ultrathin two-dimensional nanomaterials. Chem. Rev. 117, 6225-6331. doi: 10.1021/acs.chemrev.6b00558

Tang, J., Kong, B., Wu, H., Xu, M., Wang, Y., Wang, Y., et al. (2013). Carbon nanodots featuring efficient FRET for real-time monitoring of drug delivery and two-photon imaging. Adv. Mater. 25, 6569-6574. doi: 10.1002/adma.201303124

Tang, Z. H., He, C. L., Tian, H. Y., Ding, J. X., Hsiao, B. S., Chu, B., et al. (2016). Polymeric nanostructured materials for biomedical applications. Prog. Polym. Sci. 60, 86-128. doi: 10.1016/j.progpolymsci.2016.05.005

Tasis, D., Tagmatarchis, N., Bianco, A., Prato, M. (2006). Chemistry of carbon nanotubes. Chem. Rev. 106, 1105-1136. doi: 10.1021/cr050569o

Teston, E., Maldiney, T., Marangon, I., Volatron, J., Lalatonne, Y., Motte, L., et al. (2018). Nanohybrids with magnetic and persistent luminescence properties for cell labeling, tracking, in vivo real-time imaging, and magnetic vectorization. Small 14:1800020. doi: 10.1002/smll.201800020

Tian, G., Zhang, X., Gu, Z., Zhao, Y. (2015). Recent advances in upconversion nanoparticles-based multifunctional nanocomposites for combined cancer therapy. Adv. Mater. 27, 7692-7712. doi: 10.1002/adma.201503280

Tinwala, H., and Wairkar, S. (2019). Production, surface modification and biomedical applications of nanodiamonds: a sparkling tool for theranostics. Mat. Sci. Eng. C-Mater. 97, 913-931. doi: 10.1016/j.msec.2018.12.073

Tong, R., Tang, L., Ma, L., Tu, C. L., Baumgartner, R., Cheng, J. J. (2014). Smart chemistry in polymeric nanomedicine. Chem. Soc. Rev. 43, 6982-7012. doi: 10.1039/C4CS00133H

Topete, A., Alatorre-Meda, M., Iglesias, P., Villar-Alvarez, E. M., Barbosa, S., Costoya, J. A., et al. (2014). Fluorescent drug-loaded, polymeric-based, branched gold nanoshells for localized multimodal therapy and imaging of tumoral cells. ACS Nano 8, 2725-2738. doi: 10.1021/nn406425h

Treguer-Delapierre, M., Majimel, J., Mornet, S., Duguet, E., Ravaine, S. (2008). Synthesis of non-spherical gold nanoparticles. Gold Bull. 41, 195-207. doi: $10.1007 / \mathrm{BF} 03216597$

Turkevich, J., Stevenson, P. C., Hillier, J. (1951). A study of the nucleation and growth processes in the synthesis of colloidal gold. Discuss. Faraday Soc. 11, 55-75. doi: 10.1039/df9511100055

Tzur-Balter, A., Gilert, A., Massad-Ivanir, N., Segal, E. (2013). Engineering porous silicon nanostructures as tunable carriers for mitoxantrone dihydrochloride. Acta Biomater. 9, 6208-6217. doi: 10.1016/j.actbio.2012.12.010
Vaidya, A., Agarwal, A., Jain, A., Agrawal, R. K., Jain, S. K. (2011). Bioconjugation of polymers: a novel platform for targeted drug delivery. Curr. Pharm. Design 17, 1108-1125. doi: 10.2174/138161211795656873

van der Stam, W., Berends, A. C., de Mello Donega, C. (2015). Prospects of colloidal copper chalcogenide nanocrystals. Chemphyschem 17, 559-581. doi: $10.1002 /$ cphc. 201500976

Vivero-Escoto, J. L., Huxford-Phillips, R. C., Lin, W. B. (2012). Silica-based nanoprobes for biomedical imaging and theranostic applications. Chem. Soc. Rev. 41, 2673-2685. doi: 10.1039/c2cs15229k

Wang, C., Cheng, L., Liu, Z. (2013a). Upconversion nanoparticles for photodynamic therapy and other cancer therapeutics. Theranostics 3, 317-330. doi: 10.7150/thno. 5284

Wang, C. F., Makila, E. M., Kaasalainen, M. H., Liu, D. F., Sarparanta, M. P., Airaksinen, A. J., et al. (2014). Copper-free azide-alkyne cycloaddition of targeting peptides to porous silicon nanoparticles for intracellular drug uptake. Biomaterials 35, 1257-1266. doi: 10.1016/j.biomaterials.2013.10.065

Wang, J., Li, J. L., Yu, J. N., Zhang, H. W., Zhang, B. B. (2018). Large hollow cavity luminous nanoparticles with near-infrared persistent luminescence and tunable sizes for tumor afterglow imaging and chemo-/photodynamic therapies. ACS Nano 12, 4246-4258. doi: 10.1021/acsnano.7b07606

Wang, K., Fan, X., Zhao, L., Zhang, X., Zhang, X., Li, Z., et al. (2016a). Aggregation induced emission fluorogens based nanotheranostics for targeted and imaging-guided chemo-photothermal combination therapy. Small 12, 6568-6575. doi: 10.1002/smll.201601473

Wang, L. H. V., and Hu, S. (2012). Photoacoustic tomography: in vivo imaging from organelles to organs. Science 335, 1458-1462. doi: $10.1126 /$ science. 1216210

Wang, M., and Thanou, M. (2010). Targeting nanoparticles to cancer. Pharmacol. Res. 62, 90-99. doi: 10.1016/j.phrs.2010.03.005

Wang, S., Lin, J., Wang, T. F., Chen, X. Y., Huang, P. (2016b). Recent advances in photoacoustic imaging for deep-tissue biomedical applications. Theranostics 6 , 2394-2413. doi: 10.7150/thno.16715

Wang, S. J., Teng, Z. G., Huang, P., Liu, D. B., Liu, Y., Tian, Y., et al. (2015a). Reversibly extracellular $\mathrm{pH}$ controlled cellular uptake and photothermal therapy by PEGylated mixed-charge gold nanostars. Small 11, 1801-1810. doi: $10.1002 / \mathrm{smll} .201403248$

Wang, X., Wang, C., Cheng, L., Lee, S. T., Liu, Z. (2012). Noble metal coated single-walled carbon nanotubes for applications in surface enhanced Raman scattering imaging and photothermal therapy. J. Am. Chem. Soc. 134, 7414-7422. doi: 10.1021/ja300140c

Wang, X. D., Valiev, R. R., Ohulchanskyy, T. Y., Agren, H., Yang, C. H., Chen, G. Y. (2017). Dye-sensitized lanthanide-doped upconversion nanoparticles. Chem. Soc. Rev. 46, 4150-4167. doi: 10.1039/C7CS00053G

Wang, Y., Yan, B., Chen, L. (2013b). SERS tags: novel optical nanoprobes for bioanalysis. Chem. Rev. 113, 1391-1428. doi: 10.1021/cr300120g

Wang, Y., Yang, T., Ke, H. T., Zhu, A. J., Wang, Y. Y., Wang, J. X., et al. (2015b). Smart albumin-biomineralized nanocomposites for multimodal imaging and photothermal tumor ablation. Adv. Mater. 27, 3874-3882. doi: 10.1002/adma.201500229

Wang, Y. X., Feng, L. H., Wang, S. (2019). Conjugated polymer nanoparticles for imaging, cell activity regulation, and therapy. Adv. Func. Mater. 29:1806818. doi: $10.1002 / \mathrm{adfm} .201806818$

Warner, J. H., Hoshino, A., Yamamoto, K., Tilley, R. D. (2005). Water-soluble photoluminescent silicon quantum dots. Angew. Chem. Int. Ed. 44, 4550-4554. doi: 10.1002/anie.200501256

Wen, J., Yang, K., Liu, F. Y., Li, H. J., Xu, Y. Q., Sun, S. G. (2017). Diverse gatekeepers for mesoporous silica nanoparticle based drug delivery systems. Chem. Soc. Rev. 46, 6024-6045. doi: 10.1039/C7CS00219J

Wolfbeis, O. S. (2015). An overview of nanoparticles commonly used in fluorescent bioimaging. Chem. Soc. Rev. 44, 4743-4768. doi: 10.1039/C4CS00392F

Wong, P. T., and Choi, S. K. (2015). Mechanisms of drug release in nanotherapeutic delivery systems. Chem. Rev. 115, 3388-3432. doi: $10.1021 /$ cr5004634

Wu, C. F., Hansen, S. J., Hou, Q. O., Yu, J. B., Zeigler, M., Jin, Y. H., et al. (2011b). Design of highly emissive polymer dot bioconjugates for in vivo tumor targeting. Angew. Chem., Int. Ed. 50, 3430-3434. doi: 10.1002/anie.201007461

Wu, C. F., Jin, Y. H., Schneider, T., Burnham, D. R., Smith, P. B., Chiu, D. T. (2010). Ultrabright and bioorthogonal labeling of cellular targets using semiconducting 
polymer dots and click chemistry. Angew. Chem. Int. Ed. 49, 9436-9440. doi: $10.1002 /$ anie.201004260

Wu, E. C., Andrew, J. S., Cheng, L. Y., Freeman, W. R., Pearson, L., Sailor, M. J. (2011a). Real-time monitoring of sustained drug release using the optical properties of porous silicon photonic crystal particles. Biomaterials 32, 1957-1966. doi: 10.1016/j.biomaterials.2010.11.013

Wu, W., Mao, D., Xu, S., Ji, S., Hu, F., Ding, D., et al. (2017a). High performance photosensitizers with aggregation-induced emission for imageguided photodynamic anticancer therapy. Mater. Horiz. 4, 1110-1114. doi: 10.1039/C7MH00469A

Wu, W. B., Mao, D., Hu, F., Xu, S. D., Chen, C., Zhang, C. J., et al. (2017b). A highly efficient and photostable photosensitizer with near-infrared aggregationinduced emission for image-guided photodynamic anticancer therapy. $A d v$. Mater. 29:1700548. doi: 10.1002/adma.201700548

Wu, X., Chen, G. Y., Shen, J., Li, Z. J., Zhang, Y. W., Han, G. (2015). Upconversion nanoparticles: a versatile solution to multiscale biological imaging. Bioconjug. Chem. 26, 166-175. doi: 10.1021/bc5003967

Wu, Y., Ali, M. R. K., Chen, K. C., Fang, N., El-Sayed, M. A. (2019). Gold nanoparticles in biological optical imaging. Nano Today 24, 120-140. doi: 10.1016/j.nantod.2018.12.006

Xia, Y., Li, W., Cobley, C. M., Chen, J., Xia, X., Zhang, Q., et al. (2011). Gold nanocages: from synthesis to theranostic applications. Acc. Chem. Res. 44, 914-924. doi: 10.1021/ar200061q

Xu, G. X., Zeng, S. W., Zhang, B. T., Swihart, M. T., Yong, K. T., Prasad, P. N. (2016). New generation cadmium-free quantum dots for biophotonics and nanomedicine. Chem. Rev. 116, 12234-12327. doi: $10.1021 /$ acs.chemrev.6b00290

Xu, J., Murata, D., Ueda, J., Viana, B., Tanabe, S. (2018b). Toward rechargeable persistent luminescence for the first and third biological windows via persistent energy transfer and electron trap redistribution. Inorg. Chem. 57, 5194-5203. doi: 10.1021/acs.inorgchem.8b00218

Xu, S. D., Yuan, Y. Y., Cai, X. L., Zhang, C. J., Hu, F., Liang, J., et al. (2015). Tuning the singlet-triplet energy gap: a unique approach to efficient photosensitizers with aggregation-induced emission (AIE) characteristics. Chem. Sci. 6, 5824-5830. doi: 10.1039/C5SC01733E

Xu, Y. H., Wang, X. X., Zhang, W. L., Lv, F., Guo, S. J. (2018a). Recent progress in two-dimensional inorganic quantum dots. Chem. Soc. Rev. 47, 586-625. doi: $10.1039 / \mathrm{C} 7 \mathrm{CS} 00500 \mathrm{H}$

Xue, X. D., Zhao, Y. Y., Dai, L. R., Zhang, X., Hao, X. H., Zhang, C. Q., et al. (2014). Spatiotemporal drug release visualized through a drug delivery system with tunable aggregation-induced emission. Adv. Mater. 26, 712-717. doi: 10.1002/adma.201302365

Xue, Z. L., Li, X. L., Li, Y. B., Jiang, M. Y., Liu, H. R., Zeng, S. J., et al. (2017). $\mathrm{X}$-ray-activated near-infrared persistent luminescent probe for deep-tissue and renewable in vivo bioimaging. ACS Appl. Mater. Interfaces 9, 22132-22142. doi: $10.1021 /$ acsami.7b03802

Yan, C., Tian, Q., Yang, S. (2017). Recent advances in the rational design of copper chalcogenide to enhance the photothermal conversion efficiency for the photothermal ablation of cancer cells. RSC Adv. 7, 37887-37897. doi: 10.1039/C7RA05468H

Yan, X., Niu, G., Lin, J., Jin, A. J., Hu, H., Tang, Y., et al. (2015). Enhanced fluorescence imaging guided photodynamic therapy of sinoporphyrin sodium loaded graphene oxide. Biomaterials 42, 94-102. doi: 10.1016/j.biomaterials.2014.11.040

Yang, D. M., Ma, P. A., Hou, Z. Y., Cheng, Z. Y., Li, C. X., Lin, J. (2015a). Current advances in lanthanide ion $(\operatorname{Ln}(3+))$-based upconversion nanomaterials for drug delivery. Chem. Soc. Rev. 44, 1416-1448. doi: 10.1039/C4CS00155A

Yang, J., Choi, J., Bang, D., Kim, E., Lim, E. K., Park, H., et al. (2011). Convertible organic nanoparticles for near-infrared photothermal ablation of cancer cells. Angew. Chem. Int. Ed. 50, 441-444. doi: 10.1002/anie.201005075

Yang, J., Liu, Y. X., Zhao, Y. Y., Gong, Z., Zhang, M., Yan, D. T., et al. (2017a). Ratiometric afterglow nanothermometer for simultaneous in situ bioimaging and local tissue temperature sensing. Chem. Mater. 29, 8119-8131. doi: 10.1021/acs.chemmater.7b01958

Yang, K., Feng, L., Shi, X., Liu, Z. (2013). Nano-graphene in biomedicine: theranostic applications. Chem. Soc. Rev. 42, 530-547. doi: $10.1039 / \mathrm{C} 2 \mathrm{CS} 35342 \mathrm{C}$
Yang, K., Xu, H., Cheng, L., Sun, C. Y., Wang, J., Liu, Z. (2012). In vitro and in vivo near-infrared photothermal therapy of cancer using polypyrrole organic nanoparticles. Adv. Mater. 24, 5586-5592. doi: 10.1002/adma.201202625

Yang, K., Yang, G. B., Chen, L., Cheng, L., Wang, L., Ge, C. C., et al. (2015b). FeS nanoplates as a multifunctional nano-theranostic for magnetic resonance imaging guided photothermal therapy. Biomaterials 38, 1-9. doi: 10.1016/j.biomaterials.2014.10.052

Yang, S. T., Cao, L., Luo, P. G., Lu, F., Wang, X., Wang, H., et al. (2009). Carbon dots for optical imaging in vivo. J. Am. Chem. Soc. 131, 11308-11309. doi: $10.1021 / j a 904843 x$

Yang, T., Tang, Y. A., Liu, L., Lv, X. Y., Wang, Q. L., Ke, H. T., et al. (2017b). Size-dependent $\mathrm{Ag}_{2} \mathrm{~S}$ nanodots for second near-infrared fluorescence/photoacoustics imaging and simultaneous photothermal therapy. ACS Nano 11, 1848-1857. doi: 10.1021/acsnano.6b07866

Yao, J., Li, P. F., Li, L., Yang, M. (2018). Biochemistry and biomedicine of quantum dots: from biodetection to bioimaging, drug discovery, diagnostics, and therapy. Acta Biomater. 74, 36-55. doi: 10.1016/j.actbio.2018.05.004

Yin, W. Y., Yan, L., Yu, J., Tian, G., Zhou, L. J., Zheng, X. P., et al. (2014). High-throughput synthesis of single-layer $\mathrm{MoS}_{2}$ nanosheets as a near-infrared photothermal-triggered drug delivery for effective cancer therapy. ACS Nano 8, 6922-6933. doi: $10.1021 / \mathrm{nn} 501647 \mathrm{j}$

Yong, K. T. (2012). Quantum dots for biophotonics. Theranostics 2, 629-630. doi: $10.7150 /$ thno. 4757

Yu, X. H., Gao, D., Gao, L. Q., Lai, J. H., Zhang, C. R., Zhao, Y., et al. (2017). Inhibiting metastasis and preventing tumor relapse by triggering host immunity with tumor-targeted photodynamic therapy using photosensitizer-loaded functional nanographenes. ACS Nano 11, 10147-10158. doi: 10.1021/acsnano.7b04736

Yu, Z. Z., Liu, B., Pan, W., Zhang, T. T., Tong, L. L., Li, N., et al. (2018). A simple approach for glutathione functionalized persistent luminescence nanoparticles as versatile platforms for multiple in vivo applications. Chem. Commun. 54, 3504-3507. doi: 10.1039/C8CC00743H

Yuan, Y. Y., Zhang, C. J., Gao, M., Zhang, R. Y., Tang, B. Z. (2015). Specific light-up bioprobe with aggregation-induced emission and activatable photoactivity for the targeted and image-guided photodynamic ablation of cancer cells. Angew. Chem. Int. Ed. 54, 1780-1786. doi: 10.1002/anie.201408476

Zeng, P., Wei, X. T., Yin, M., Chen, Y. H. (2018). Investigation of the long afterglow mechanism in $\mathrm{SrAl}_{2} \mathrm{O}_{4}: \mathrm{Eu}^{2+} / \mathrm{Dy}^{3+}$ by optically stimulated luminescence and thermoluminescence. J. Lumin. 199, 400-406. doi: 10.1016/j.jlumin.2018.03.088

Zhan, D. D., Liu, J. M., Song, N., Liu, Y. Y., Dang, M., Fang, G. Z., et al. (2018). Fabrication of mesoporous $\mathrm{La}_{3} \mathrm{Ga}_{5} \mathrm{GeO}_{14}: \mathrm{Cr}^{3+}, \mathrm{Zn}^{2+}$ persistent luminescence nanocarriers with super-long afterglow for bioimaging-guided in vivo drug delivery to the gut. J. Mater. Chem. 6, 1479-1488. doi: 10.1039/C7TB02759A

Zhang, L. W., Rong, P. F., Chen, M. L., Gao, S., Zhu, L. (2015). A novel single walled carbon nanotube (SWCNT) functionalization agent facilitating in vivo combined chemo/thermo therapy. Nanoscale 7, 16204-16213. doi: $10.1039 /$ C5NR03752B

Zhang, S. H., Sun, C. X., Zeng, J. F., Sun, Q., Wang, G. L., Wang, Y., et al. (2016). Ambient aqueous synthesis of ultrasmall PEGylated $\mathrm{Cu}_{2-\mathrm{X}} \mathrm{Se}$ nanoparticles as a multifunctional theranostic agent for multimodal imaging guided photothermal therapy of cancer. Adv. Mater. 28, 8927-8936. doi: 10.1002/adma.201602193

Zhang, W., Jiang, L. M., Piper, J. A., Wang, Y. L. (2018). SERS nanotags and their applications in biosensing and bioimaging. J. Anal. Test. 2, 26-44. doi: 10.1007/s41664-018-0053-9

Zhang, Y. S., Wang, Y., Wang, L. D., Wang, Y. C., Cai, X., Zhang, C., et al. (2013). Labeling human mesenchymal stem cells with gold nanocages for in vitro and in vivo tracking by two-photon microscopy and photoacoustic microscopy. Theranostics 3, 532-543. doi: 10.7150/thno.5369

Zhao, P., Xu, Q., Tao, J., Jin, Z. W., Pan, Y., Yu, C. M., et al. (2018). Near infrared quantum dots in biomedical applications: current status and future perspective. Wiley Int. Rev. 10:e1483. doi: 10.1002/wnan.1483

Zhao, P. Q., Ji, W., Zhou, S. Y., Qiu, L. H., Li, L. F., Qian, Z. Z., et al. (2017). Upconverting and persistent luminescent nanocarriers for accurately imagingguided photothermal therapy. Mater. Sci. Eng. 79, 191-198. doi: 10.1016/j.msec.2017.05.046 
Zhong, J. P., Wen, L. W., Yang, S. H., Xiang, L. Z., Chen, Q., Xing, D. (2015). Imaging-guided high-efficient photoacoustic tumor therapy with targeting gold nanorods. Nanomedicine 11, 1499-1509. doi: 10.1016/j.nano.2015.04.002

Zhou, J., Liu, Z., Li, F. Y. (2012). Upconversion nanophosphors for small-animal imaging. Chem. Soc. Rev. 41, 1323-1349. doi: 10.1039/C1CS15187H

Zhou, M., Tian, M., Li, C. (2016b). Copper-based nanomaterials for cancer imaging and therapy. Bioconjugate Chem. 27, 1188-1199. doi: 10.1021/acs.bioconjchem.6b00156

Zhou, Z. J., Song, J. B., Nie, L. M., Chen, X. Y. (2016a). Reactive oxygen species generating systems meeting challenges of photodynamic cancer therapy. Chem. Soc. Rev. 45, 6597-6626. doi: 10.1039/C6CS00271D

Zhu, H. J., Fang, Y., Zhen, X., Wei, N., Gao, Y., Luo, K. Q., et al. (2016). Multilayered semiconducting polymer nanoparticles with enhanced NIR fluorescence for molecular imaging in cells, zebrafish and mice. Chem. Sci. 7, 5118-5125. doi: 10.1039/C6SC $01251 \mathrm{E}$
Zou, R., Gong, S. M., Shi, J. P., Jiao, J., Wong, K. L., Zhang, H. W., et al. (2017) Magnetic-NIR persistent luminescent dual-modal ZGOCS@MSNs@ $\mathrm{Gd}_{2} \mathrm{O}_{3}$ core-shell nanoprobes for in vivo imaging. Chem. Mater. 29, 3938-3946. doi: 10.1021/acs.chemmater.7b00087

Conflict of Interest: The authors declare that the research was conducted in the absence of any commercial or financial relationships that could be construed as a potential conflict of interest.

Copyright (c) 2019 Yang, Wang, Wan, Gu and Li. This is an open-access article distributed under the terms of the Creative Commons Attribution License (CC BY).

The use, distribution or reproduction in other forums is permitted, provided the original author(s) and the copyright owner(s) are credited and that the original publication in this journal is cited, in accordance with accepted academic practice. No use, distribution or reproduction is permitted which does not comply with these terms. 